\title{
Particle tracking in drug and gene delivery research: State-of-the-art applications and methods ${ }^{2}$
}

\author{
Benjamin S. Schuster ${ }^{\text {a,b, 1,2 }}$, Laura M. Ensign ${ }^{\text {a,c,e,2, }}$, Daniel B. Allan ${ }^{\text {d }}$, Jung Soo Suk ${ }^{\text {a,c }}$, Justin Hanes ${ }^{\text {a,b,c,e,* }}$ \\ a Center for Nanomedicine, Johns Hopkins University School of Medicine, Baltimore, MD 21231, USA \\ b Department of Biomedical Engineering, Johns Hopkins University School of Medicine, Baltimore, MD 21205, USA \\ c Department of Ophthalmology, Johns Hopkins University School of Medicine, Baltimore, MD 21231, USA \\ d Department of Physics and Astronomy, Johns Hopkins University, Baltimore, MD 21218, USA \\ e Department of Chemical \& Biomolecular Engineering, Johns Hopkins University, Baltimore, MD 21218, USA
}

\section{A R T I C L E I N F O}

Available online $\mathrm{xxxx}$

\section{Keywords:}

Automated particle tracking

Nanomedicine

Nanoparticle

Biological barrier

Mucus

Diffusion

Microscopy

Image analysis

\begin{abstract}
A B S T R A C T
Particle tracking is a powerful microscopy technique to quantify the motion of individual particles at high spatial and temporal resolution in complex fluids and biological specimens. Particle tracking's applications and impact in drug and gene delivery research have greatly increased during the last decade. Thanks to advances in hardware and software, this technique is now more accessible than ever, and can be reliably automated to enable rapid processing of large data sets, thereby further enhancing the role that particle tracking will play in drug and gene delivery studies in the future. We begin this review by discussing particle tracking-based advances in characterizing extracellular and cellular barriers to therapeutic nanoparticles and in characterizing nanoparticle size and stability. To facilitate wider adoption of the technique, we then present a user-friendly review of state-ofthe-art automated particle tracking algorithms and methods of analysis. We conclude by reviewing technological developments for next-generation particle tracking methods, and we survey future research directions in drug and gene delivery where particle tracking may be useful.
\end{abstract}

(c) 2015 Elsevier B.V. All rights reserved.

\section{Contents}

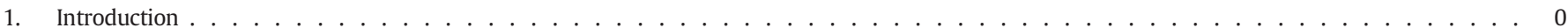

2. The utility of particle tracking analysis in drug and gene delivery . . . . . . . . . . . . . . . . . . . . . . . . . . . . 0

2.1. Particle tracking to assess nanoparticle size and stability in complex biological environments . . . . . . . . . . . . . . . . . . . . 0

2.2. Particle tracking to characterize extracellular barriers to drug and gene delivery . . . . . . . . . . . . . . . . . . . 0

2.2.1. Particle tracking in the vitreous . . . . . . . . . . . . . . . . . . . . . . . . . . . . . . . 0

2.2.2. Particle tracking in the brain extracellular matrix $(\mathrm{ECM}) \quad \ldots \ldots \ldots \ldots$

2.2.3. Particle tracking in reconstituted mucin gels . . . . . . . . . . . . . . . . . . . . . . . . 0

2.2.4. Particle tracking in human cervicovaginal mucus . . . . . . . . . . . . . . . . . . . . . . . . . . . 0

2.2.5. Particle tracking in animal gastrointestinal and cervicovaginal mucus . . . . . . . . . . . . . . . . . . . . . 0

2.2.6. Particle tracking of nanoparticles in biofilms and human $\mathrm{CF}$ sputum . . . . . . . . . . . . . . . . . . . . . 0

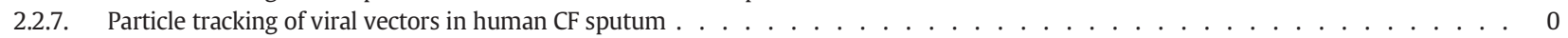

2.2.8. Particle tracking of non-viral vectors in human CF sputum ～. . . . . . . . . . . . . . . . . . . . . . . . 0

2.2.9. Particle tracking in human respiratory mucus . . . . . . . . . . . . . . . . . . . . . . . . . . . . . . . . . . 0

2.3. Particle tracking to characterize cellular barriers to drug and gene delivery . . . . . . . . . . . . . . . . . . . . . . . . 0

2.3.1. Membrane and cytoplasmic dynamics of therapeutic nanoparticles . . . . . . . . . . . . . . . . . . . . . . . 0

2.3.2. Simultaneous tracking of nanoparticles and cellular compartments . . . . . . . . . . . . . . . . . . . . . . . . . . 0

2.3.3. Strategies to improve cytoplasmic dynamics of therapeutic nanoparticles . . . . . . . . . . . . . . . . . . . . . . . . 0

2.3.4. Dynamics of therapeutic nanoparticles near the cell nucleus . . . . . . . . . . . . . . . . . . . . . . . 0

2.3.5. Intracellular dynamics of therapeutic nanoparticles in $3 \mathrm{D} \ldots \ldots \ldots \ldots$

\footnotetext{
This review is part of the Advanced Drug Delivery Reviews theme issue on "Editor's Choice 2015"

* Corresponding author at: Center for Nanomedicine at the Wilmer Eye Institute, Johns Hopkins University School of Medicine, 400 N. Broadway, Robert H. and Clarice Smith Building, 6th Floor, Baltimore, MD 21231, USA. Tel.: +1 410614 6513; fax: +1 4106146509. E-mail address: hanes@jhmi.edu (J. Hanes).

${ }^{1}$ Present address: Department of Bioengineering, University of Pennsylvania, Philadelphia, PA 19104, USA.

2 These authors contributed equally to this work.
} 
2.4. Broader biomedical applications of particle tracking analysis $\ldots \ldots \ldots \ldots$

2.4.1. Particle tracking as a method for probing microrheology . . . . . . . . . . . . . . . . . . . . . . . . . 0

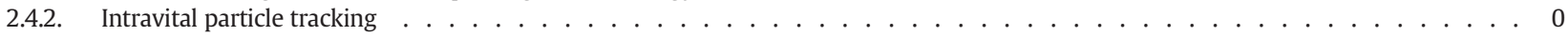

3. Implementing particle tracking in nanomedicine research: a quick-start guide . . . . . . . . . . . . . . . . . . . . . . . . 0

3.1. Design of particle tracking experiments: particles, microscope, and camera . . . . . . . . . . . . . . . . . . . . 0

3.2. Extracting quantitative measurements from particle tracking videos: automated particle tracking using the Crocker and Grier algorithm . . . 0

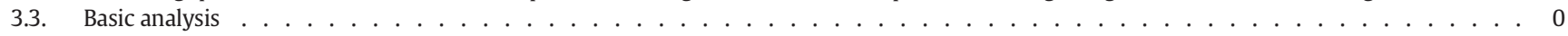

4. Advanced topics in particle tracking . . . . . . . . . . . . . . . . . . . . . . . . . . . . . . . 0

4.1. Data analysis and interpretation in "messy," heterogeneous biological environments . . . . . . . . . . . . . . . . . . . . 0

4.2. High-precision particle tracking and methods to minimize error . . . . . . . . . . . . . . . . . . . . . . . 0

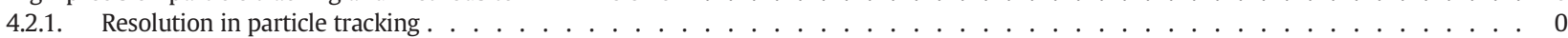

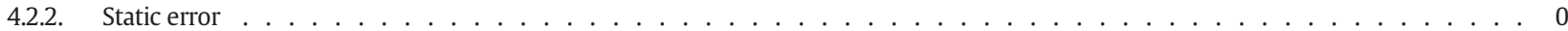

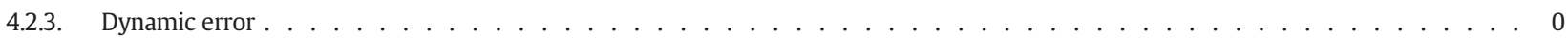

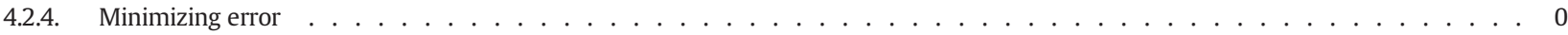

4.3. Enhanced algorithms for tracking in challenging biological environments . . . . . . . . . . . . . . . . . . . . . . . . 0

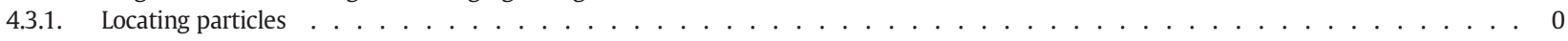

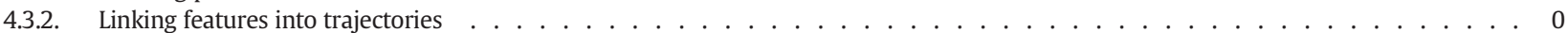

4.4. Advanced microscopy techniques for particle tracking in thick specimens . . . . . . . . . . . . . . . . . . . . . 0

4.5. Computational tools for reproducible and high-throughput particle tracking . . . . . . . . . . . . . . . . . . . . . . . . . 0

5. Outlook: future applications of particle tracking to drug and gene delivery research . . . . . . . . . . . . . . . . . . . . . . . . . . . . . 0

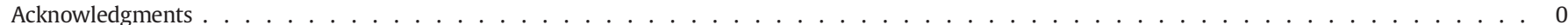

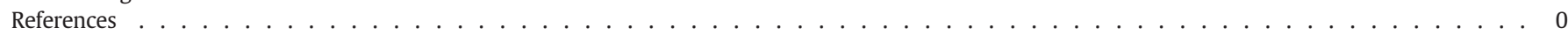

\section{Introduction}

Nanoparticle (NP) based drug and gene carriers often must overcome biological barriers in order to achieve therapeutic efficacy in target cells, tissues, and organs. Depending on the site of administration, NPs usually must remain stable in, and transport through, extracellular barriers [1-4] before potentially being taken up by cells [5], and in some cases, must traffic through the cell to the nucleus [6,7] or other subcellular organelles [8]. Particle tracking is a powerful technique for studying the motion of particles at high spatial and temporal resolution, allowing quantification of transport rates of thousands of individual drug carriers, imaging agents, synthetic gene vectors, and viruses through cells, tissues, and biological fluids. Particle tracking is also a valuable tool for characterizing the microscopic structure and rheology of biological specimens [9], and for determining NP size and stability in biological fluids [10]. Compared to techniques that measure average values over entire ensembles of particles, tracking with single-particle resolution permits advanced analysis of heterogeneous particle behavior and fluid characteristics.

Particle tracking has matured over the past three decades into a broadly accessible technique that is useful in a variety of fields. Early applications were in cellular biophysics [11,12] and fundamental colloid science [13]. During the past 15 years, our lab and others have harnessed the power of particle tracking for drug and gene delivery research. Particle tracking experiments can be conducted with microscopes and modern cameras that are now standard in biomedical research facilities. Numerous software packages for analysis of particle tracking videos are now freely available, and they can perform well even when studying complex biological specimens. Users do not require much, if any, prior programming experience to process even large data sets.

In the decade since our last review of particle tracking for drug and gene delivery [14], particle tracking has been adapted to probe numerous additional barriers and has enabled significant discoveries that have guided more effective drug and gene delivery. In the following section, we review these advances. To facilitate widespread adoption of this powerful technique, we then present a user-friendly review of state-of-the-art particle tracking methods, and discuss how to minimize errors to achieve tracking that is quantitatively precise and qualitatively correct. We conclude with an outlook on future directions in the field.

\section{The utility of particle tracking analysis in drug and gene delivery}

NP design criteria can vary greatly depending on the cargo (e.g. drug, protein, nucleic acid) and the target site for delivery. Particle tracking can be used to characterize NP systems facing common design challenges, such as: (1) NPs delivering nucleic acids to cells should remain stable during transit, but become unstable enough to release the cargo upon reaching the intracellular environment; (2) NPs with targeting functionality should have affinity for the target, but not bind to off-target molecules, nor bind so tightly to the target that the particle is not released into the intracellular environment; (3) NPs may not need to be internalized at all, but may need to overcome other biological barriers to deliver drugs locally to tissues. Below, we discuss particle tracking studies designed to characterize various NP systems in diverse biological fluids for a wide range of drug and gene delivery applications.

\subsection{Particle tracking to assess nanoparticle size and stability in complex biological environments}

NP size, size distribution, and morphology have a significant impact on NP biodistribution, clearance, and cell uptake. The most common methods for characterization of NP size distribution include dynamic light scattering (DLS) and electron microscopy, though other techniques such as size exclusion chromatography, gel electrophoresis, asymmetric flow field-flow fractionation, and analytical ultracentrifugation have also been used [15-17]. Of all these techniques, DLS is perhaps the most common, as it is user-friendly and yields results in a relatively short period of time using automated machines. However, DLS has drawbacks that are inherent to the technique itself [18]. NP size is determined from fluctuations in the intensity of scattered light due to the Brownian motion of the particles in solution. The intensity of the scattered light is proportional to the sixth power of the particle diameter [19], so the measurements are weighted toward the presence of large particles, including aggregates and dust. In contrast, particle tracking methods identify and track individual NPs, rather than calculating distributions based on entire particle populations. Thus, nanoparticle tracking analysis (NTA) was developed and made commercially available with the NanoSight device in 2006. The NanoSight device provides an automated, integrated system that more rapidly characterizes samples with minimal user effort. However, both the NanoSight and DLS are limited to measurements in simple media and diluted biological fluids. Whether intended for intravenous (IV) injection or local administration, NPs encounter various proteins that can coat their surface, alter their interactions with target cells and tissues, and potentially induce aggregation. Protein adsorption can also impact NP clearance rates and biodistribution [20-23]. Thus, while NPs are often characterized in simple 
buffers and solvents, it is important to study NP size and aggregation in undiluted biological fluids.

To address the limitations of DLS and the NanoSight in characterizing NP size and aggregation in undiluted biological fluids, Braeckmans and coworkers adapted fluorescence particle tracking microscopy [10]. They injected fluorescent liposomes into the bloodstream of rats, collected blood samples over time, and used a microscope to observe the diffusion of the liposomes in the blood samples (Fig. 1). The trajectories were used to estimate diffusion coefficients, which were then transformed to size distributions using a maximum entropy deconvolution method (MEM). They made liposomes composed of commercially available lipids (DOTAP, DOPE), as well as formulations containing polyethylene glycol conjugated lipids (DSPE-PEG, ceramide-PEG). Significant aggregation of the DOTAP-DOPE liposomes was observed in blood collected 5 min after IV administration, leading to a decrease in the number of intact liposomes in the blood samples over time. In contrast, liposomes with ceramide-PEG incorporated had a limited amount of aggregates that were eliminated at later time points, and liposomes with DSPE-PEG incorporated were more stable in the blood for the 60 min measurement period. Importantly, particle tracking in whole blood revealed liposome instability that was not evident using standard characterization techniques in aqueous solutions or in separated serum and plasma [10].

Stability is also a major concern for NPs formed through ionic interactions to deliver cargo such as small interfering RNA (siRNA) or other nucleic acids, as exposure to biological environments can cause the particles to dissociate in vivo. Naeye and coworkers used particle tracking to assess the stability and biodistribution of covalently cross-linked dextran nanogels (dex-NG) that can be loaded with siRNA via electrostatic interactions [24]. Previous work by this group demonstrated that addition of $5 \mathrm{kDa}$ PEG to the surface of siRNA-loaded dex-NG reduced interactions with blood cells and prevented aggregation in human plasma [25]. Similarly, Naeye found that the PEGylated dex-NG were stable upon incubation in mouse blood and plasma, yet the clearance after IV administration of free siRNA, siRNA-loaded dex-NG, and PEGylated siRNA-loaded dex-NG was indistinguishable [24]. Using particle tracking, they discovered that although the complexes were stable upon incubation in isolated mouse blood, rapid aggregation of the PEGylated siRNA-loaded dex-NG occurred in systemic circulation after IV injection. The authors attributed the aggregation to insufficient or inhomogeneous PEGylation of the nanogels, leading to aggregation and rapid clearance [24]. In related work, Ragelle and coworkers described the development of chitosan-based NPs for IV delivery of siRNA [26]. To increase NP stability and provide "stealth" properties, PEG was grafted onto the chitosan ( $\mathrm{C}_{\mathrm{PEG}}$ ) and hyaluronic acid (HA) was incorporated. Particle tracking was then used to assess the stability of $\mathrm{C}_{\mathrm{PEG}} /$ polyethylenimine (PEI)/HA and $\mathrm{C}_{\mathrm{PEG}} / \mathrm{PEI}$ particles in human plasma. $\mathrm{C}_{\mathrm{PEG}} / \mathrm{PEI}$ particles rapidly aggregated, whereas the $\mathrm{C}_{\mathrm{PEG}} / \mathrm{PEI} / \mathrm{HA}$ particles remained stable for the duration of the measurement (time not specified) [26]. However, as the Naeye work emphasized, an important next step is to ascertain that the $\mathrm{C}_{\mathrm{PEG}} / \mathrm{PEI} / \mathrm{HA}$ siRNA particles remain stable during circulation, as opposed to in isolated plasma.

Intraperitoneal (IP) injection may be a favorable route of drug or gene carrier administration for certain indications, such as peritoneal metastases associated with ovarian or other cancers. Dakwar and coworkers used particle tracking to study the colloidal stability of polystyrene (PS) NPs and liposomes with varying surface properties in mouse IP fluid, plasma from a healthy patient, and ascites fluid from a patient diagnosed with peritoneal carcinomatosis [27]. They tested cationic (amine-modified), anionic (carboxylate-modified), and neutrally charged (coated with $2 \mathrm{kDa}$ PEG) PS NPs. In contrast to cationic and anionic particles, the PEGylated PS NPs remained stable in all three fluids, with only a minor increase in size compared to that observed in buffer. They then formulated liposomes composed of DOTAP and DOPE (DOTAP-DOPE). The cationic DOTAP-DOPE liposomes formed large aggregates $(2-3 \mu \mathrm{m}$ in size) that settled to the bottom of the well, so particle tracking measurements could not be performed. The 5\% PEGylated liposomes showed some improvement in stability, and the 10\% PEGylated liposomes appeared to be the most stable in the various fluids. However, increasing the amount of PEG reduced the cationic surface charge, and therefore decreased the amount of siRNA complexed to the particle surface [27]. This work highlights the trade-offs that must often be made when formulating NPs to have multiple functionalities, such as stability in serum, increased circulation time, and complexation of nucleic

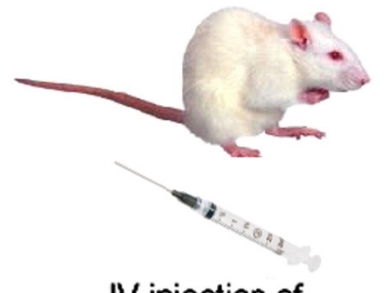

IV injection of fluorescent nanoparticles

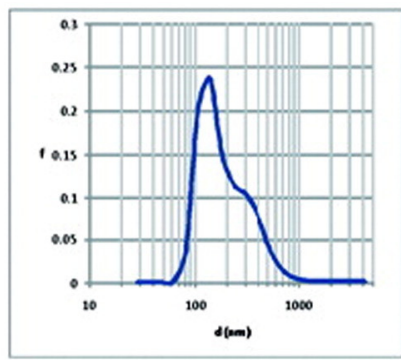

Nanoparticle size distribution (MEM analysis)

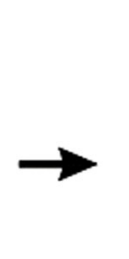

Blood sample collection

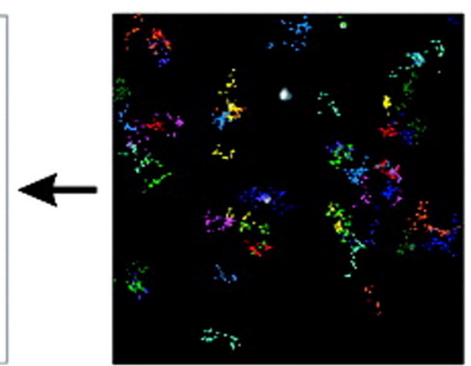

Calculate individual trajectories

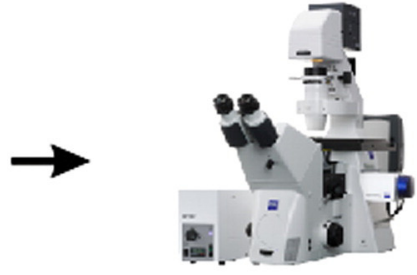

To single particle tracking microscope
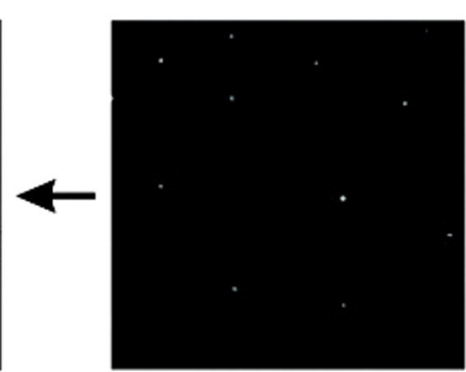

Record movie of diffusing nanoparticles

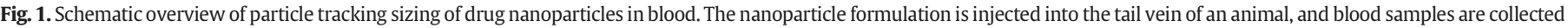

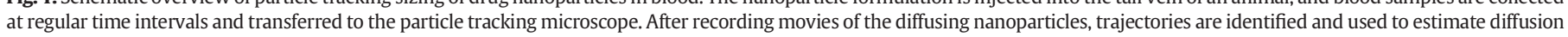
coefficients to convert to a continuous size distribution. Modified with permission from [10]. 
acids. Particle tracking is a useful and informative tool to assess the success of imparting multiple functionalities to a NP formulation in complex biological environments, such as blood and IP fluid, which has tremendous impact on the efficacy of drug and gene delivery.

\subsection{Particle tracking to characterize extracellular barriers to drug and gene delivery}

NPs may encounter numerous extracellular steric and adhesive obstacles before reaching target cells and tissues. Particle tracking measures NP interactions with, and mobility in, these biological barriers, thereby providing insight that can be used to improve the design of nanomaterials for drug and gene delivery. Although high-resolution particle tracking typically only permits observation of particle motion for relatively short times and distances, we will describe several examples in which NP transport observed by particle tracking in ex vivo samples correlated well with the distribution and penetration of biological barriers in vivo.

\subsubsection{Particle tracking in the vitreous}

Particle tracking was used recently to investigate the impact of surface chemistry on NP diffusion in vitreous fluid and to characterize the length-scale dependent rheology of the vitreous. Intravitreal injection is a promising route for NP-based drug and gene delivery to the back of the eye; depending on the application, the goal may be to inject larger particles that remain immobilized and provide sustained release of therapeutics, or to inject smaller particles that can diffuse throughout the vitreous and deliver nucleic acid therapies to the cells of the retina. $\mathrm{Xu}$ and coworkers tracked the diffusion of polystyrene (PS) NPs with cationic (PS- $\mathrm{NH}_{2}$ ), anionic (PS-COOH), and neutral (5 kDa PEG, PSPEG) surface chemistry and of various sizes ( 100 nm-1200 nm) in fresh bovine eyes that were carefully handled so as to minimize any disruption of the delicate vitreous gel [28]. Particle tracking revealed that a 2 -fold increase in hydrodynamic diameter, from $510 \mathrm{~nm}$ to $1200 \mathrm{~nm}$, led to a 30-fold decrease in the mean squared displacement (MSD) of non-adhesive PS-PEG NPs in bovine vitreous. Particle tracking microrheology calculations using the trajectories of non-adhesive PSPEG NPs revealed that the average pore size of bovine vitreous was about $550 \mathrm{~nm}$, with the presence of some pores $800 \mathrm{~nm}$ in size or larger. In contrast to PS-PEG NPs, $200 \mathrm{~nm}$ PS- $\mathrm{NH}_{2}$ NPs were adhesively immobilized in the vitreous gel. Anionic PS-COOH NPs 85 and $230 \mathrm{~nm}$ in diameter diffused freely in bovine vitreous, whereas PS-COOH NPs $500 \mathrm{~nm}$ and larger were hindered. They then went on to demonstrate that the design criteria established with PS NPs for rapid diffusion in the vitreous could be used as a guideline for formulating NPs for drug and gene delivery to cells in the back of the eye. Biodegradable NPs composed of poly(lactic-co-glycolic acid) (PLGA) and polyvinyl alcohol (PVA) (230 nm) with neutral surface charge, as well as highly compacted, neutrally charged DNA NPs composed of block copolymers of polylysine and $10 \mathrm{kDa}$ PEG $\left(\mathrm{CK}_{30} \mathrm{PEG}_{10 \mathrm{k}}\right)(60 \mathrm{~nm})$, both rapidly penetrated bovine vitreous. In contrast, cationic polyethylenimine (PEI) DNA NPs formed immobilized aggregates in the bovine vitreous [28].

Aggregation and immobilization are particularly problematic for NPs carrying DNA, as they must be able to reach and be internalized by target cells to exert their therapeutic effect. Martens and coworkers performed a similar work with bovine eyes that were incubated for $24 \mathrm{~h}$ after NP injection before particle tracking [29]. They found that $100 \mathrm{~nm}$ cationic NPs were adhesively immobilized in the bovine vitreous. They did not observe a significant hindrance of $1000 \mathrm{~nm}$ PS-PEG (PEGylated with $2 \mathrm{kDa}$ PEG) NPs, or of 500-1000 nm PS-COOH NPs, but did observe a relative hindrance in the diffusion of PS-COOH NPs compared to PS-PEG NPs of the same size, which they attributed to hydrophobic interactions with components of the vitreous. They also tested the diffusion of DNA NPs composed of linear poly(amido amine) with repetitive disulfide linkages compared to a version with $2.25 \mathrm{kDa}$ PEG on the surface. Particle tracking revealed that both the PEGylated and non-PEGylated DNA NPs diffused similarly in buffer. However, the PEGylated DNA NPs diffused more rapidly in bovine vitreous, though at a slower rate than in buffer, and there was a significant fraction of non-PEGylated DNA NPs that were adhesively immobilized [29]. Adhesively immobilized DNA NPs would be unable to reach target cells, further highlighting the valuable insight that particle tracking can provide when designing NP formulations for drug and gene delivery.

\subsubsection{Particle tracking in the brain extracellular matrix (ECM)}

The blood brain barrier (BBB) has long been considered a major barrier to effective treatment of central nervous system diseases. However, there are numerous instances in which BBB function is disrupted, including brain tumors and neuroinflammation, as well as via techniques like high-intensity focused ultrasound for reversibly disrupting the BBB [30]. When a NP crosses the BBB, or is directly infused into the brain parenchyma, such as through convection-enhanced delivery (CED), distribution throughout the brain extracellular matrix (ECM) itself is a barrier to effective drug and gene delivery [30]. It was previously suggested that NPs must be $<64 \mathrm{~nm}$ in diameter to penetrate through the brain ECM [31]. However, Nance, Woodworth, and coworkers examined whether dense coatings of low molecular weight PEG might allow larger NPs to penetrate the brain parenchyma, which would be advantageous because larger particles offer higher drug loading efficiency and longer drug release [2]. Indeed, particle tracking revealed that PEG-coated PS (PS-PEG) NPs as large as $114 \mathrm{~nm}$ in size diffused rapidly in rodent brain ECM ex vivo (freshly excised tissue sections) and in vivo (using intravital particle tracking; see Section 2.4.2) (Fig. 2). Importantly, $114 \mathrm{~nm}$ PS-PEG also rapidly penetrated through the ECM in human brain slices ex vivo. Using an obstruction scaling model and the particle tracking data for $114 \mathrm{~nm}$ PS-PEG, they estimated that pores as large as $225 \mathrm{~nm}$ existed in the human and rodent brain, with about $28 \%$ of the pores larger than $100 \mathrm{~nm}$. The dramatic difference in
A

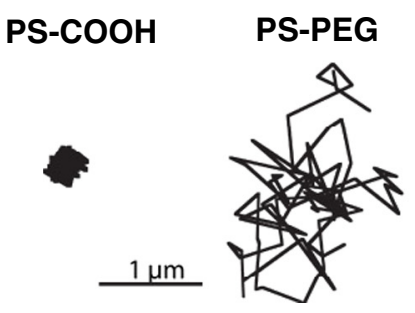

B

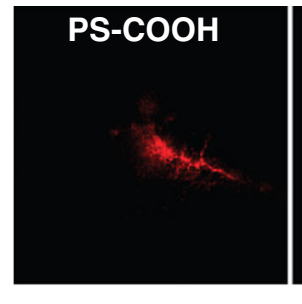

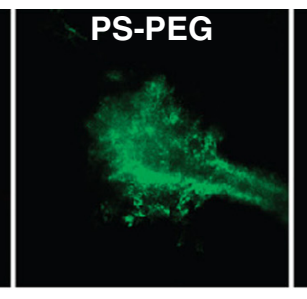

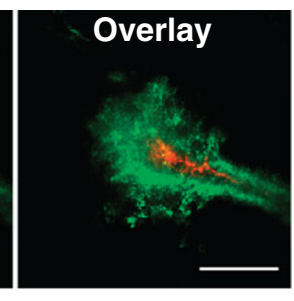

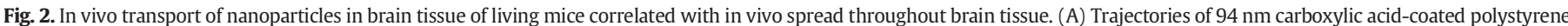

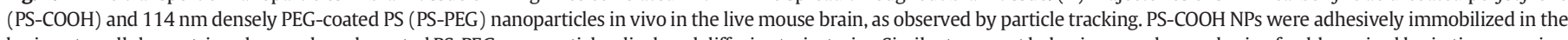

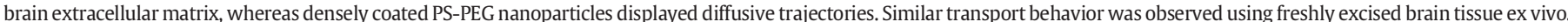

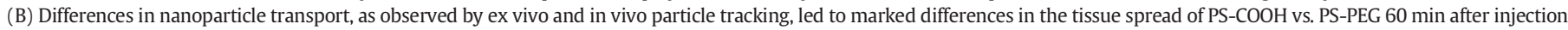

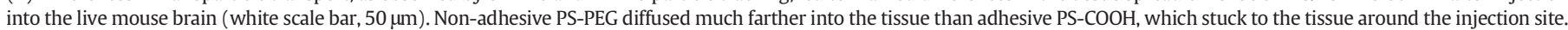
Modified with permission from [2]. 
estimated pore sizes compared to prior work could be attributed to the highly dense PEG coatings that were required to render the particles truly adhesion-resistant in the brain ECM. PS NPs and quantum dots with low density PEG coatings remained largely adhesively immobilized in brain tissue, which led to underestimation of the size of the pores in the ECM [2].

Nance and coworkers then demonstrated that so-called brainpenetrating nanoparticles (BPNs) exhibited increased distribution in brain tumors and led to improved efficacy against malignant glioma in an animal model [30]. They first used particle tracking to demonstrate that 9 L glioma tumor tissue was more restrictive to NP diffusion, due to the dramatically increased cellular density, compared to normal brain tissue. Thus, they formulated PLGA NPs densely coated with PEG, $70 \mathrm{~nm}$ in size, and loaded with paclitaxel (PTX/PLGA-PEG). These particles rapidly diffused in ex vivo rat tumor tissue, particularly in comparison to the adhesively immobilized uncoated PLGA NPs (PTX/PLGA). The non-adhesive PTX/PLGA-PEG NPs provided improved distribution compared to PTX/PLGA NPs throughout the brain tumor tissue after direct injection, leading to greater suppression of the aggressive $9 \mathrm{~L}$ gliosarcoma tumor growth. The tumor load for the PTX/PLGA NPs group at day 15 was $45 \%$ of the nontreated group, but the tumor load for the PTX/PLGA-PEG NPs was only $8 \%$ of the control group and statistically lower than any other group at day 15 [30].

Building upon this work, Schneider and coworkers designed densely PEG-coated NPs that were decorated with a monoclonal antibody that recognizes fibroblast growth factor-inducible 14 (Fn14), which is strongly expressed in high-grade malignant gliomas, yet minimally expressed in normal human brain [32]. Using particle tracking, they discovered that these Fn14-targeted PEG-coated NPs can effectively penetrate rat brain ECM, similarly to PEG-coated NPs without additional functionalization. Furthermore, the targeted particles were able to selectively bind to and be uptaken by Fn14-positive human glioblastoma cells. This provides proof of concept that it is possible to design NPs that do not bind to brain ECM proteins, and thus can diffuse within brain tissue, and that can also target brain tumor cells. The authors suggest that this is a promising approach for selectively delivering therapeutics to kill brain tumor cells outside of the area safe for surgical removal.

Mastorakos and coworkers next investigated the effects of surface properties on penetration of gene vectors in the brain ECM [33]. They formulated PEI-based DNA NPs that were uncoated (DNA-UPN), conventionally PEG coated (lower PEG density, DNA-CPN), and densely PEG coated (brain-penetrating, DNA-BPN). Using particle tracking in ex vivo rodent brain tissue, they demonstrated that DNA-UPN were largely immobilized by the brain ECM, and DNA-CPN exhibited hindered motion. In contrast, DNA-BPN diffused relatively unhindered over much larger distances in the brain ECM. At a time scale of $1 \mathrm{~s}$, DNA-BPN were on average 5- and 29-fold faster than DNA-CPN and DNA-UPN, respectively. Accordingly, DNA-BPN were able to spread over a much larger volume and much farther from the injection site in the rodent brain compared to DNA-CPN following bolus co-injection in vivo. They demonstrated an even more striking difference in distribution in the rodent brain following administration by CED - the volume of distribution for DNA-BPN was at least 3-fold higher than DNA-CPN, and the volume of transgene expression achieved by DNA-BPN in the brain was 2.4-fold higher than DNA-CPN and 3.2-fold higher than DNA-UPN. Similarly, the overall transgene expression in the brain was 2-fold higher when delivered with DNA-BPN compared to DNA-CPN [33]. These results indicate that more rapid, uniform diffusion of DNA NPs in brain ECM, as observed by particle tracking ex vivo, leads to greater distribution and delivery in vivo, similar to the findings with drug-loaded NPs.

\subsubsection{Particle tracking in reconstituted mucin gels}

Mucosal surfaces, such as those of the respiratory tract, the female reproductive tract, the gastrointestinal tract, and the surface of the eye, are important target sites for drug and gene delivery [3,34,35]. However, the viscoelastic, adhesive mucus layers lining mucosal surfaces are tenacious barriers to NPs. NPs that are adhesively or sterically trapped in mucus are unlikely to reach tissue or cellular targets, and are typically rapidly removed via natural mucus clearance mechanisms [3, 34]. Reconstituted mucin gels are often used as convenient in vitro systems for assessing NP mobility and stability in mucus, which is important for achieving effective NP-mediated drug and gene therapy and vaccination at mucosal surfaces. Dawson and coworkers formulated PLGA NPs coated with the cationic surfactant DDAB in order to condense DNA to the surface (PLGA-DDAB/DNA) [36]. Using particle tracking and reconstituted pig gastric mucin, they found some NP aggregation in mucin, likely due to the cationic DDAB coating. However, the average diffusion rate of PLGA-DDAB/DNA NPs was 10-fold higher than similarly sized PS NPs, indicating that the PLGA-DDAB/DNA NPs were generally stable and able to move in mucin solution [36].

Lieleg and coworkers used particle tracking to investigate how $\mathrm{pH}$ and ionic strength impacted PS NP diffusion in reconstituted porcine gastric mucin gels [37]. They found that diffusion of $1000 \mathrm{~nm}$ cationic (amine-modified) PS NPs were hindered by $1 \%$ mucin gels unless high ionic strength buffers were added to shield the ionic interactions. In contrast, diffusion of PEGylated PS NPs was nearly unaffected by the ionic strength of the buffer used to reconstitute the mucins, as ionic interactions were minimized by the PEG coatings. They also found that the diffusion of PEGylated PS NPs was significantly influenced by the mucin concentration of the reconstituted mucin gel, due to steric effects, and by the $\mathrm{pH}$. The $\mathrm{pH}$ may affect the interactions between the mucins and charged particles, and it may also affect the gel microstructure [37]. This work highlights the physiochemical complexity of mucus and the utility of particle tracking in characterizing mucus barrier properties.

\subsubsection{Particle tracking in human cervicovaginal mucus}

Although particle tracking in reconstituted mucin gels can provide insight into the nanoscale interactions between NPs and mucins, tracking NPs in fresh, undiluted mucus secretions is more informative and relevant as regards expected NP behavior in vivo. Lai and coworkers demonstrated with particle tracking that fresh, undiluted human cervicovaginal mucus (CVM) from women with healthy vaginal microbiota is a significant adhesive barrier to conventional, hydrophobic NPs, such as PS [1]. In contrast, PS NPs coated with a high density of $2 \mathrm{kDa}$ PEG (PS-PEG), and as large as $530 \mathrm{~nm}$, diffused in CVM only a few times slower than their theoretical diffusivity in water [1]. Later work used particle tracking to demonstrate the importance of PEG density in minimizing adhesion to CVM [38], the design of PLGA-PEG block copolymer NPs that rapidly penetrate CVM [39], and the physical adsorption of PEG layers onto NP surfaces to produce NPs that rapidly penetrate CVM [40].

However, particle tracking has been particularly useful in resolving non-uniform NP transport behavior. Tang and coworkers described the formulation of NPs composed of biodegradable block copolymers of poly(sebacic acid) and PEG (PSA-PEG) [41]. Using particle tracking, they observed that PSA-PEG NPs uniformly penetrate CVM, but found that there were significant populations of hindered and immobile NPs in human cystic fibrosis (CF) sputum. However, the other 40\% of PSAPEG NPs diffused relatively rapidly in CF sputum [41]. This suggested that the fluid contained within the pores of CF sputum only had slightly higher viscosity than the fluid in the CVM pores, but the majority of the pores in CF sputum were smaller in diameter. Methods that provide bulk transport averages may have led to the incorrect conclusion that the overall average NP MSD was reduced due to significantly increased viscosity of the fluid contained within the pores in CF sputum. Similarly, Ensign and coworkers observed that pretreatment of CVM with Pluronic F127 led to diffusion of typically mucoadhesive 200 nm PS NPs [42]. However, in contrast to the uniformly diffusive PS-PEG NPs with dense, covalently conjugated [43] or physically adsorbed [40] PEG coatings, 
only 50\% of the PS NPs were diffusive in Pluronic F127 pretreated CVM [42]. Thus, particle tracking revealed that there were two distinct populations of diffusive and adhesive NPs, whereas the lower overall average MSD measurement could have been mistaken for an overall increase in CVM viscosity due to the Pluronic pretreatment.

2.2.5. Particle tracking in animal gastrointestinal and cervicovaginal mucus

Although it is ideal to test NP systems for diffusion in fresh, minimally-diluted human mucus secretions, it is also important to test NP behavior in the animal models in which efficacy and pharmacokinetics are initially studied. Additionally, animal mucus can be used as a model system for difficult to obtain mucus secretions, such as gastrointestinal (GI) mucus. Macierzanka and coworkers obtained proximal (jejunal) small intestine mucus from both piglets and adult pigs to investigate how microstructure and permeability are impacted by age of the animal and extracellular DNA content [44]. Mucus was scraped from the intestines of pigs after slaughter, and then frozen and stored at $-80^{\circ} \mathrm{C}$ until use. Particle tracking revealed that $500 \mathrm{~nm}$ carboxylate-modified PS NPs were immobile in pig mucus, but when the mucus was pretreated with DNase, $64 \%$ of the PS NPs were freely diffusive. In piglet mucus, $30 \%$ of the $500 \mathrm{~nm}$ PS NPs were diffusive, whereas $77 \%$ were freely diffusive after mucus pretreatment with DNase. They concluded that the porcine small intestine mucus contained DNA derived from small intestine epithelial cells that contributes to mucus viscoelastic properties. They also suggested that the difference in adhesivity of $500 \mathrm{~nm}$ PS NPs in adult pig vs. piglet mucus observed by particle tracking implied differences in cell turnover or distribution of mucin-producing goblet cells during postnatal development compared to adulthood [44].

The processing methods and sources of material must always be considered when interpreting particle tracking data obtained with biological hydrogels, such as mucus. In order to address the limitation of obtaining fresh and minimally perturbed mucus secretions from areas such as the GI tract or the airways, Ensign and coworkers developed an ex vivo technique for characterizing particle transport by particle tracking in mucus secretions on freshly excised tissue [45]. Using conventional (mucoadhesive) uncoated polystyrene particles (CP) and densely PEGylated mucus-penetrating particles (MPP), they first demonstrated that the quantitative particle tracking results obtained using mouse colorectal mucus gently removed from the tissue surface were not different from the results obtained when visualizing the NPs in mucus on top of freshly excised tissue mounted in a custom well. Using the freshly excised ex vivo tissue, they observed that only MPP $110 \mathrm{~nm}$ or less in diameter diffuse rapidly in mouse colorectal mucus, whereas MPP as large as $210 \mathrm{~nm}$ could rapidly diffuse in mouse small intestine mucus. The difference in the apparent mucus pore structure at these two anatomical sites may reflect the difference in function (nutrient absorption in the small intestine and water absorption/exclusion of bacteria in the colorectum [46]). In contrast, $\mathrm{CP}$ of all sizes were adhesively immobilized in both mouse colorectal and small intestine mucus. Particle tracking also revealed a significant difference in the transport of similarly sized MPP in the vaginal mucus of mice under different hormonal influence. High estradiol levels during the estrus phase resulted in more rapid diffusion of MPP, whereas high progestin levels associated with pretreatment with Depo Provera (DP) resulted in more hindered diffusion of MPP, reflecting the influence of hormones on vaginal mucus pore structure [45].

Importantly, Ensign and coworkers demonstrated a strong correlation between more rapid particle transport measured by particle tracking ex vivo and more uniform distribution of NPs over the mucosal surface in vivo; when particle tracking revealed rapid, uniform diffusion of NPs in ex vivo mouse estrus vaginal mucus, nearly uniform distribution of MPP along the vaginal mucosal surface was observed in vivo. In contrast, the hindered diffusion of MPP in DP-treated mouse vaginal mucus ex vivo was reflected in non-uniform distribution throughout the mucus in vivo, restricting the NPs from reaching the epithelial surface [45]. A similar result was demonstrated in other work by Ensign and

coworkers, where they demonstrated near uniform vaginal distribution of NPs in vivo that could rapidly diffuse in mouse vaginal mucus, as observed by particle tracking ex vivo (Fig. 3) [3]. In contrast, NPs that were adhesively immobilized in mouse vaginal mucus ex vivo, as observed by particle tracking, were found aggregated in the vaginal lumen in vivo, and were restricted from reaching the vaginal epithelial surface [3].

\subsubsection{Particle tracking of nanoparticles in biofilms and human CF sputum}

Airway mucus plays a significant role in both health and disease. Particularly in the case of obstructive airway diseases, such as cystic fibrosis $(\mathrm{CF})$, airway mucus is a barrier to effective pulmonary treatment. CF is a disease that can be treated by gene therapy in theory, yet the CF sputum coating the airways of CF patients is a formidable barrier to gene therapy in the lungs [47]. Suk and coworkers used particle tracking to probe the local microstructure of freshly expectorated human CF sputum, as experienced on the nanoscale by amine-modified PS (PS- $\left.\mathrm{NH}_{2}\right)$ NPs and densely PEG-coated (PEG-PS) NPs [48]. Particle tracking revealed that $200 \mathrm{~nm}$ PS- $\mathrm{NH}_{2}$ NPs were adhesively immobilized in CF sputum, confirming prior results [49], whereas a fraction of $200 \mathrm{~nm}$ PEG-PS NPs diffused, and the movement of $500 \mathrm{~nm}$ PEG-PS NPs was largely hindered. An obstruction scaling model, initially developed to model the diffusion of non-interacting solutes in hydrogels [50], was used to calculate an average mesh spacing in CF sputum of $140 \pm 50 \mathrm{~nm}$ [48]. The average pore size in CF sputum was significantly lower than the $340 \pm$ $70 \mathrm{~nm}$ average pore size that was previously estimated for CVM using particle tracking data obtained using non-adhesive NPs and the same obstruction scaling model [43]. This work again highlighted the power of particle tracking techniques for characterizing the structural and barrier properties of biological fluids to NPs intended for use in drug and gene delivery.

Hill and coworkers demonstrated in vitro that particle tracking using large PS NPs can be used to measure changes in mucus viscoelasticity,
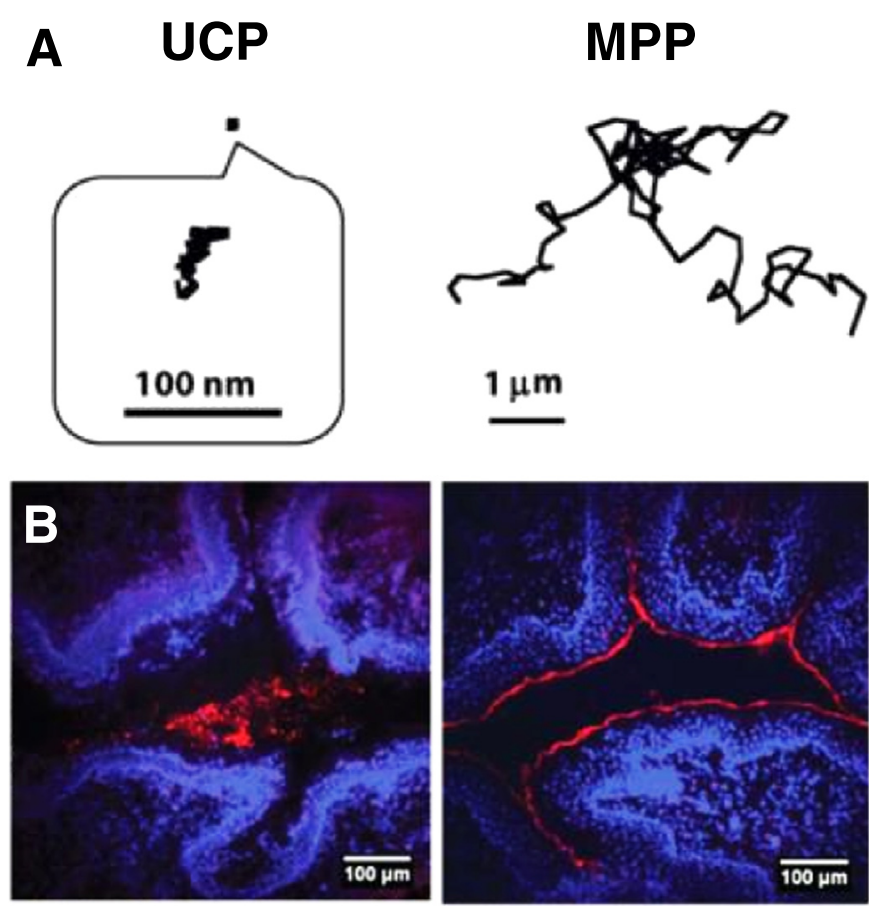

Fig. 3. Ex vivo transport of $100 \mathrm{~nm} N P$ in mouse cervicovaginal mucus (CVM) correlates with in vivo distribution in the mouse vagina. (A) Trajectories (3 s of motion) of uncoated (UCP) and densely PEG-coated (MPP) nanoparticles in mucus on freshly excised ex vivo mouse vaginal tissue, as observed by particle tracking. UCP remained adhesively immobilized in mouse CVM, whereas MPP displayed diffusive trajectories. (B) Mucoadhesive UCP were trapped in the luminal mucus, whereas MPP penetrated through the mucus to distribute over nearly the entire tissue surface within $10 \mathrm{~min}$. The differences in nanoparticle transport, as observed by particle tracking, led to marked differences in the cervicovaginal distribution of UCP and MPP. Modified with permission from [3]. 
demonstrating that increases in mucus solid concentration can be used as a candidate biomarker for airway disease [51]. As diseases such as CF progress, solid content in airway mucus secretions increases, leading to further reductions in mesh spacing and overall increase in the barrier properties of CF sputum to NPs. This makes gene delivery more challenging, whether viral or non-viral gene vectors are used, and exacerbates symptoms such as impaired mucociliary clearance [51]. This work also highlighted the potential for particle tracking as a tool for characterizing the progression of diseases, such as $\mathrm{CF}$.

Forier and coworkers tested the structural heterogeneity in CF sputum samples from three separate patients using particle tracking with 100 and $200 \mathrm{~nm}$ cationic, anionic, and PEGylated PS NPs (both $2 \mathrm{kDa}$ and $5 \mathrm{kDa}$ PEG) [52]. Their particle tracking measurements confirmed that charged NPs are more hindered, with a higher fraction of immobilized NP, in CF sputum, whereas PEG-coated NPs diffuse more rapidly. However, the $\mathrm{CF}$ sputum from one of the patients even immobilized a significant portion of $100 \mathrm{~nm}$ PEG-coated NPs, and essentially all $200 \mathrm{~nm}$ PEG-coated NPs, indicating that this patient's sputum had a very tight mesh structure. They also used particle tracking to investigate the interactions of the various NP types with bacterial biofilm cultures. CF patients suffer from impaired mucociliary clearance and chronic bacterial infection and biofilm formation on the surface of the epithelium [53]. The authors observed that carboxylate-modified PS NPs, which were largely hindered in CF sputum, appeared to accumulate in the outer edges of the bacterial clusters and closely associate with the bacteria themselves [52]. Thus, they concluded that a NP formulation that somehow combined the sputum-penetrating properties of PEG-coated particles and the direct interaction with biofilms observed for anionic NPs may be ideal for delivering antibiotics in the CF airways [52].
Messiaen and coworkers then used particle tracking to evaluate tobramycin-loaded liposomes for treating Burkholderia cepacia biofilms [54]. Generally, they found that anionic and cationic PS NPs exhibited restricted diffusion through various types of Burkholderia biofilms, which they attributed to electrostatic interactions with biofilm components. They next prepared tobramycin liposomes composed of commercially available DPPC, DOPC, and DPPG lipids; DPPC and cholesterol liposomes were neutrally charged, while DPOC/DPPG liposomes had anionic surface charge. However, neither the anionic nor neutral tobramycin-loaded liposomes had increased bactericidal activity compared to free tobramycin in vitro [54]. It is possible that the larger size of the neutral liposomes ( $>400 \mathrm{~nm}$ ) restricted their diffusion in the biofilms, which could be evaluated with fluorescent labeling of the liposomes and particle tracking. This study highlights the utility of particle tracking in designing nanoparticle-based therapy strategies for penetrating and more effectively delivering drugs to biofilms.

\subsubsection{Particle tracking of viral vectors in human CF sputum}

Based on the estimated average pore size of CF sputum of $\sim 140 \mathrm{~nm}$ [48], viral gene vectors such as adenovirus ( $\mathrm{AdV}, \sim 80 \mathrm{~nm}$ ) and adenoassociated virus (AAV) serotype $5(\sim 20 \mathrm{~nm})$ are small enough to penetrate through the CF sputum gel to reach the periciliary layer next to the airway epithelium. However, Hida and coworkers used particle tracking to demonstrate that these viral vectors, which have been extensively studied for CF gene therapy, are adhesively immobilized in CF sputum and, thus, cannot penetrate the sputum gel layer [55]. In contrast to much larger (200 nm) PEG-coated NPs that diffused in CF sputum at rates only 40 -fold slowed compared to their theoretical diffusivity in pure water, AdV and AAV5 moved 3000 and 12,000 fold more slowly in CF sputum [55]. Adhesion of viral vectors to CF sputum has likely

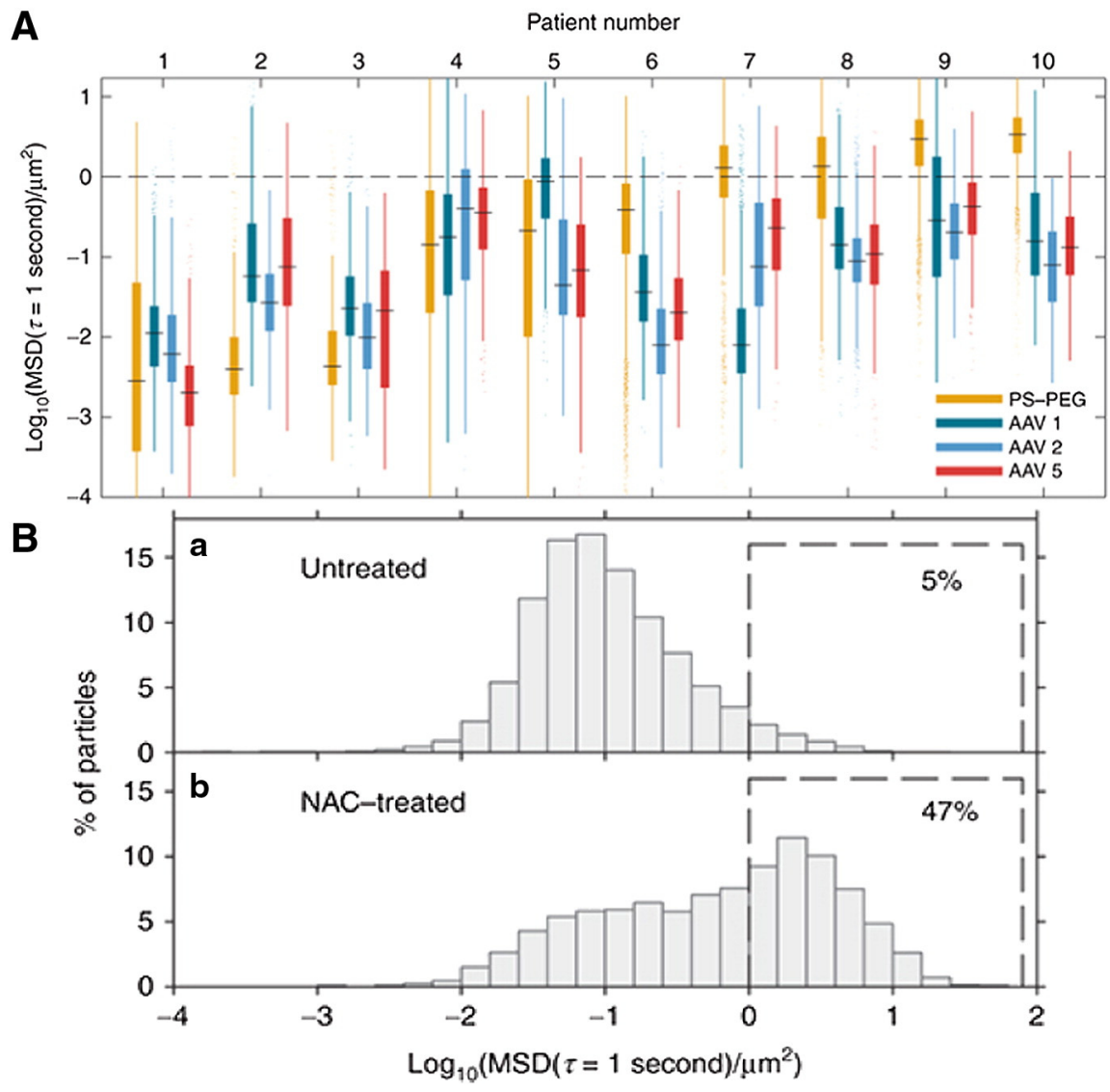

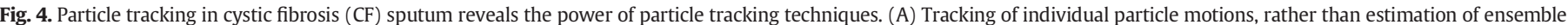

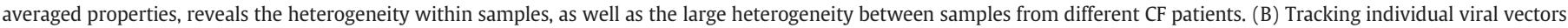

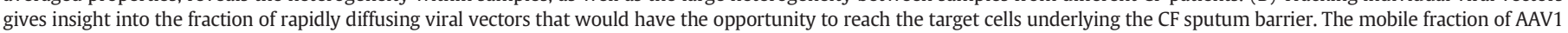
in CF sputum was increased from $5 \%$ to $47 \%$ by pretreating sputum with a mucolytic (N-acetylcysteine, NAC). Modified with permission from [57]. 
contributed to the poor outcomes observed in CF gene therapy clinical trials to date [56].

To address this issue, Schuster and coworkers used particle tracking to explore techniques for increasing penetration of viral vectors through CF sputum [57]. Particle tracking revealed significant patient-to-patient variability in the permeability of CF sputum to viruses and to NPs similar in size to viruses (Fig. 4A). They typically found that PEGylated PS NPs, though larger in size than AAV, diffused more rapidly in CF sputum than did the viruses. Moreover, 50\% of AAV1, AAV2, and AAV5 diffused $>1000$-fold slower in sputum than in water. They then tested two strategies for improving viral vector penetration through $\mathrm{CF}$ sputum. A mutant AAV2 engineered for reduced heparin binding diffused 2-fold faster than native AAV2 in CF sputum, presumably due to reduced adhesion. They also discovered that a mucolytic (N-acetylcysteine, NAC) could alter sputum structure and thereby reduce viral adhesion; only $5 \%$ of AAV1 rapidly diffused in CF sputum before treatment of CF sputum

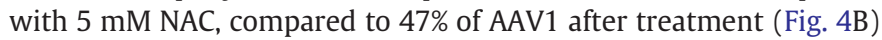
[57]. This result was consistent with prior work demonstrating that NAC improves the mobility of NPs in CF sputum [58]. Both approaches, generating novel AAV mutants and pretreating CF sputum with mucolytics, have promise for improving viral CF gene therapy.

\subsubsection{Particle tracking of non-viral vectors in human CF sputum}

Due to the immunogenicity and genome size limitations associated with viral gene vectors, there is also a strong need for the development of efficient non-viral gene delivery systems. The only polymeric nonviral system that has been tested in clinical trials for $\mathrm{CF}$ is a polylysine polymer conjugated to $10 \mathrm{kDa}$ PEG via a cysteine residue ( $\left.\mathrm{CK}_{30} \mathrm{PEG}_{10 \mathrm{k}}\right)$. DNA compacted into small rods or ellipsoids using $\mathrm{CK}_{30} \mathrm{PEG}_{10 \mathrm{k}}$ $\left(\mathrm{CK}_{30} \mathrm{PEG}_{10 \mathrm{k}}\right.$ DNA NPs) mediated high levels of gene transfer to the human nasal mucosa and was safe [59], but it was never demonstrated to be effective in the CF lung airways. Boylan and coworkers used particle tracking to determine whether $\mathrm{CK}_{30}$ systems with varying PEG molecular weights $(2,5,10 \mathrm{kDa})$ could penetrate freshly expectorated CF sputum [60]. All three DNA NP systems were stable, resisted digestion by DNase $\mathrm{I}$, and mediated comparable gene transfer in the lungs of mice. However, particle tracking revealed that $\mathrm{CK}_{30} \mathrm{PEG}_{2 \mathrm{k}}$ DNA NPs, $\mathrm{CK}_{30} \mathrm{PEG}_{5 \mathrm{k}}$ DNA NPs, and $\mathrm{CK}_{30} \mathrm{PEG}_{10 \mathrm{k}}$ DNA NPs were all adhesively immobilized in CF sputum, likely due to insufficient surface PEG density [60].

Suk and coworkers tested whether mucolytic treatments could improve the diffusion of $\mathrm{CK}_{30} \mathrm{PEG}_{10 \mathrm{k}}$ DNA NPs in CF sputum [58]. CF sputum contains both high amounts of DNA from lysed cells, as well as high concentrations of mucins. Using particle tracking, they discovered that pretreatment of CF sputum with rhDNase alone had no impact on the restricted diffusion of $\mathrm{CK}_{30} \mathrm{PEG}_{10 \mathrm{k}}$, but mucolytic NAC pretreatment and combination rhDNase/NAC pretreatment lead to an increase in the percentage of $\mathrm{CK}_{30} \mathrm{PEG}_{10 \mathrm{k}}$ DNA NPs diffusing in $\mathrm{CF}$ sputum. They then used ex vivo tracking with freshly excised mouse tracheal mucosa [45] to demonstrate that pulmonary NAC pretreatment of mice challenged with lipopolysaccharide (LPS) to induce mucus hypersecretion led to an increase in the diffusive fraction of $\mathrm{CK}_{30} \mathrm{PEG}_{10 \mathrm{k}}$ DNA NPs in the airway mucus. As a further demonstration of the correlation between particle tracking observations and in vivo efficacy, they showed that NAC pretreatment also strongly increased gene transfection mediated by $\mathrm{CK}_{30} \mathrm{PEG}_{10 \mathrm{k}}$ DNA NPs in the airways of mice with LPS-induced mucus hypersecretion [58].

More recently, polymeric gene vectors with higher PEG surface density were described that are capable of penetrating CF sputum without pretreatment with mucolytics or enzymes [4,61]. Kim and coworkers described a system based on cystamine core poly(amido amine) (PAMAM S-S) dendrimers [4]. The exterior branches of the dendrimer were grafted with PEG, and then the core sulfhydryl linkage was conjugated to polycationic polymers, such as PEI, for DNA condensation. Particle tracking analysis revealed that the PEGylated dendrimer-DNA (dPEG-PAMAM/DNA) NPs diffused in freshly expectorated CF sputum, whereas non-PEGylated PAMAM/DNA NPs were adhesively immobilized [4]. Suk and coworkers then demonstrated with particle tracking that various branching and polymer blending strategies can be used to make DNA NPs with high enough PEG surface density to diffuse in CF sputum while still stably compacting DNA [61]. PEI DNA NPs without PEG coatings (PEI-UCP) were adhesively immobilized in freshly expectorated CF sputum. In contrast, a significant portion of densely PEG-coated PEI DNA NPs (PEI-MPP) and PLL DNA NPs (PLL-MPP) diffused in CF sputum. In good agreement with the improved diffusion of PEI-MPP in CF sputum observed by particle tracking ex vivo (Fig. 5A), PEI-MPP exhibited improved airway distribution following inhalation into mice (Fig. 5B), prolonged lung retention in mice compared to PEI$\mathrm{UCP}$, and improved gene transfection in the airways of mice in vivo (Fig. $5 \mathrm{C}$ ) [61].

\subsubsection{Particle tracking in human respiratory mucus}

Respiratory mucus in the healthy airways is also a substantial barrier to NPs, including viruses. Collecting airway secretions for particle tracking is very challenging, so mucus from animals is often used as a model for human mucus secretions. Yang and coworkers used particle tracking to characterize the barrier properties of porcine tracheal mucus to NPs and pseudorabies virus (PRV), a virus that infects the porcine upper respiratory tract [62]. They observed that the diffusion of cationic and anionic PS NPs was obstructed in porcine tracheal mucus. In
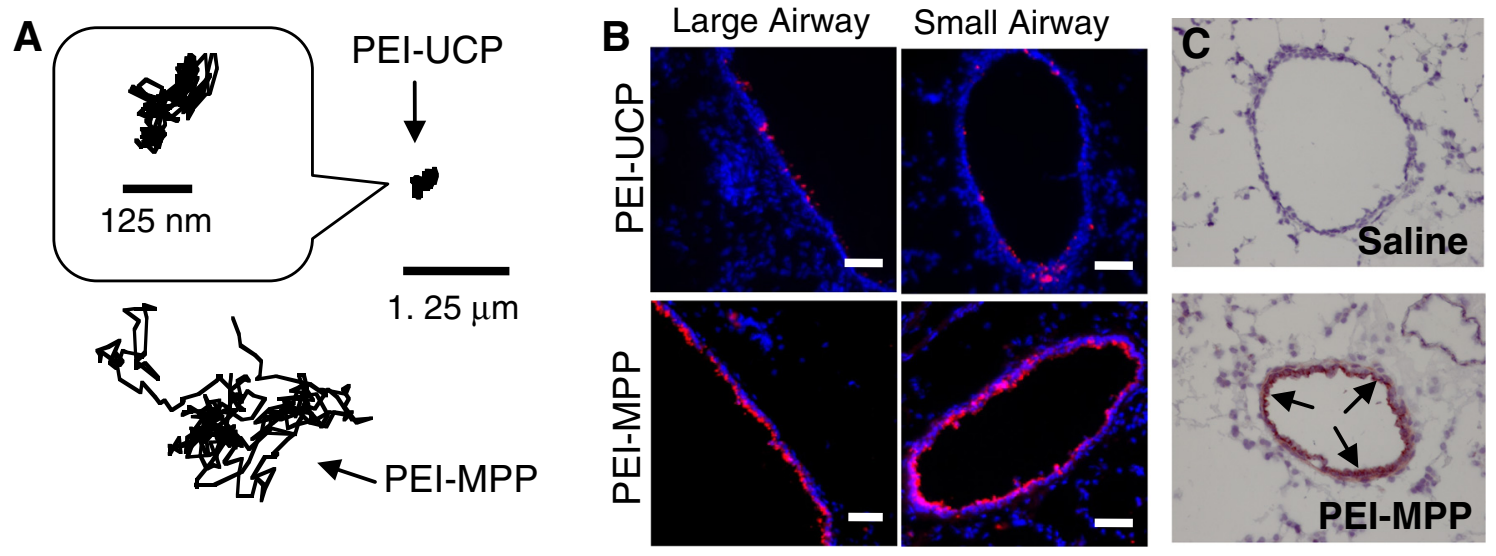

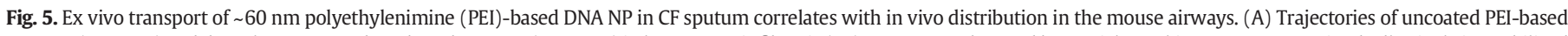

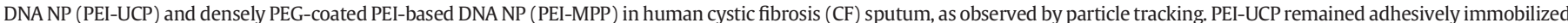

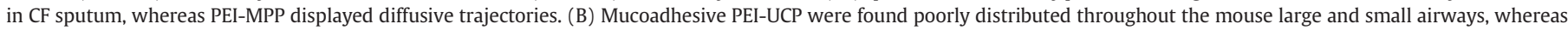

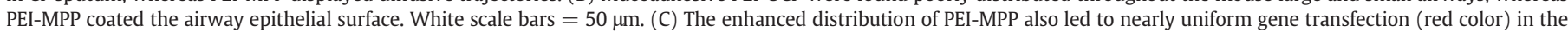
mouse airways in vivo. Modified with permission from [61]. 
contrast, PEG-coated NPs diffused rapidly in porcine tracheal mucus, so the diffusion of PEG-coated 100, 200, and $500 \mathrm{~nm}$ PS NPs as observed by particle tracking was used to estimate the pore size distribution, which was found to include pores ranging from $\sim 100-1300 \mathrm{~nm}$ in diameter. Despite the relatively large size of the mucus pores compared to the size of PRV ( $250 \mathrm{~nm})$, the diffusion of PRV was obstructed. PEGylated NPs with a hydrodynamic diameter of $\sim 233 \mathrm{~nm}$ diffused 160 -fold faster than PRV, indicating that PRV must be interacting adhesively with the porcine tracheal mucus [62]. This observation is consistent with previous particle tracking observations that found that human immunodeficiency virus (HIV) and herpes simplex virus type 1 (HSV) must be adhesively immobilized in CVM from women with healthy vaginal microbiota, as larger PEGylated NPs were able to rapidly diffuse in CVM $[43,63]$. Yang and coworkers also tested whether PEGylating the surface of PRV would decrease its adhesive interactions with mucus, and found that surface PEGylation increased the diffusion of PRV in porcine tracheal mucus by 11 -fold [62].

Schuster and coworkers recently described a method for collecting airway mucus from humans without lung disease, which they used to perform particle tracking analysis of NP transport [64]. In their method, endotracheal tubes from surgical patients with no respiratory comorbidities were removed and centrifuged to collect airway mucus secretions. They found that 100 and $200 \mathrm{~nm}$ PEG-coated (PS-PEG) NPs were able to diffuse in human respiratory mucus, whereas $500 \mathrm{~nm}$ PSPEG NPs were sterically trapped in the mucus mesh. This result was somewhat surprising based on the scanning electron microscope image of human respiratory mucus they obtained, which appeared to contain a majority of pores $<100 \mathrm{~nm}$ in size. Such an observation further emphasizes the importance of using particle tracking to directly observe how NPs interact with biological fluids. Interestingly, carboxyl-modified PS (PS-COOH) NPs of 200 and $500 \mathrm{~nm}$ in size were immobilized, but $40 \%$ of $100 \mathrm{~nm}$ PS-COOH NPs were diffusive in human respiratory mucus. The authors hypothesized that the endogenous surfactant present in respiratory mucus may coat hydrophobic NPs, making small particles that form fewer contacts with the mesh around them less adhesive [64]. The particle tracking methods described here with human respiratory mucus can be applied to design and test novel therapeutic NP systems for airway delivery in disease states such as lung cancer.

\subsection{Particle tracking to characterize cellular barriers to drug and gene delivery}

NPs that successfully overcome extracellular barriers to reach the vicinity of target cells encounter additional cellular barriers. For example, gene vectors carrying plasmid DNA must overcome the cellular membrane, degradative endosomes, cytoplasm, and the nuclear envelope for the DNA payload to be delivered into the nucleus for transcription [65]. Drug-loaded NPs, particularly those delivering certain chemodrugs, may need to enter cells and escape endosomes in order to bypass drug resistance mechanisms and achieve therapeutically relevant intracellular drug concentrations [66].

\subsubsection{Membrane and cytoplasmic dynamics of therapeutic nanoparticles}

A variety of techniques, including flow cytometry and various static microscopy methodologies, have been employed to study endocytic mechanisms and subcellular localization of NPs; however, the tracking of individual particles using particle tracking allows for direct observation of the internalization and intracellular dynamics of NPs at each step. Seisenberger and coworkers adopted single particle tracking to investigate the infection kinetics of AAV (serotype not specified), in live cells [7]. They showed that AAV labeled with a single Cy5 molecule contacted the cell membrane multiple times, with a mean contact time of $62 \mathrm{~ms}$, before diffusing away or penetrating into the cells. They found that only $13 \%$ of the tracked AAV was endocytosed, underscoring the cell membrane as a significant barrier to gene delivery. Once inside cells, AAV exhibited three different transport modes: hindered diffusion, normal diffusion, and microtubule (MT)-dependent directed motion toward the nucleus [7]. The MT-dependent transport of various viruses has been suggested based on molecular biology techniques [67, 68] and/or simple time-lapse microscopy [69], but quantitatively confirmed for the first time in this study. They also found that $50 \%$ of the tracked AAV was found in the nucleus 15 min after the initiation of the experiment, which was much faster than the $2 \mathrm{~h}$ infection time estimated previously [70]. The authors argued that conventional methods require accumulation of a higher number of AAV for detection, thus leading to overestimation of the infection time [7].

It was previously believed that the efficiency of non-viral gene vectors reaching the peri-nuclear region of cells after internalization was dramatically reduced compared to viral vectors, due to the relatively slow random diffusion of non-viral vectors in the cytoplasm $[71,72]$. However, using multiple particle tracking, Suh and coworkers demonstrated that a widely studied non-viral gene vector, polyethylenimine (PEI)/DNA NPs, can also traffic via MT-dependent active transport at a similar rate to motor proteins (i.e. dyneins and kinesins) on MTs, in addition to more traditional Brownian and hindered diffusion [6]. The authors observed perinuclear accumulation of PEI/DNA NPs within $30 \mathrm{~min}$, similar to the previously determined time required for perinuclear accumulation of adenovirus (AdV). The dependence on MTs for active transport of PEI/DNA NPs was confirmed by the abrogation of active transport upon treating cells with a MT depolymerizing agent, nocodazole [6]. Similarly, Xiao and coworkers demonstrated using particle tracking that AdV and herpes simplex virus (HSV) move bidirectionally on MTs, whereas AAV serotype 2 (AAV2) rapidly moves unidirectionally toward the nucleus [73]. Suk and coworkers directly compared intracellular transport and trafficking of PEI/DNA NPs and AdV in primary neurons, using particle tracking and confocal microscopy [74]. Similar fractions of both viral and non-viral vectors demonstrated active transport at comparable rates. However, PEI/DNA NPs were primarily sequestered within endosomes, whereas most of the AdV were found in the cytoplasm, in good agreement with a previous finding that AdV efficiently escapes endosomes [75]. Likewise, AdV has been shown to actively transport on MTs after escaping endosomes [76]. Kulkarni and coworkers reported that b-cyclodextrin (b-CD)/DNA NPs within endosomes underwent MT-mediated active transport at short time scales $(t<7 \mathrm{~s})$, but exhibited subdiffusive motions at longer time scales, which they attributed to thermal bending modes of the MT networks [77]. They concluded that b-CD/DNA NPs were localized within endosomes based on previously observed dynamics of endosomes, motor proteins, and MTs.

\subsubsection{Simultaneous tracking of nanoparticles and cellular compartments}

By combining live-cell confocal microscopy and dual-color particle tracking, Suh and coworkers simultaneously traced the transport of vesicles of the endo-lysosomal pathway (in cells expressing fluorescent versions of proteins known to localize in these vesicles) and PEI/DNA NPs [78]. They found that PEI/DNA NPs were actively transported within both endosomes and late endosomes/lysosomes, confirming the previous findings in neurons [74]. Interestingly, despite the widely supported hypothesis that PEI/DNA NPs can efficiently escape endosomes, they observed that PEI/DNA NPs transferred between late endosomes/ lysosomes but did not detect escape into the cytoplasm [78]. The lack of observed endosomal escape may indicate that longer observation times would be needed to observe PEI/DNA NP escape, or that perhaps only small amounts of scattered PEI/DNA NPs with low fluorescent intensity escaped but were not visible in comparison to the high intensity of PEI/DNA NPs sequestered in endosomes. Bausinger and coworkers also have demonstrated intracellular transport of PEI/DNA NPs within endosomes [79]. Using particle tracking, they identified three sequential transport modes: free diffusion, restricted diffusion up to the point of complete immobilization, and active transport. They postulated that the small fraction exhibiting free diffusion was PEI/DNA NPs undergoing lateral diffusion on the cellular membrane, similar to DOTAP/DOPE and 
DOTAP/DOPC lipoplexes shown to undergo lateral motion on the lipid bilayer prior to the membrane fusion [80]. The population diffusing in a restricted manner was thought to be PEI/DNA NPs undergoing engulfment by the cellular membrane via actin filaments [79]. They also observed active transport of PEI/DNA along actin filaments with velocities similar to those reported for engulfed glycoprotein-binding beads on actin filaments [80]. At later times ( $t>90 \mathrm{~min}$ ), PEI/DNA NPs exhibited long-range directed motions with increased velocities. They simultaneously monitored PEI/DNA NPs and MTs to directly confirm that PEI/DNA NPs were actively transported on MTs, supporting the prior observation that nocodazole-induced MT disruption ceased active transport [6]. They also found that 95\% of PEI/DNA NPs remained in endosomes for $>180 \mathrm{~min}$ and exhibited bidirectional motions [79], in good agreement with previous findings [78]. Similar to the PEI/DNA NPs, epidermal growth factor receptor (EGFR)-targeted PEI/DNA NPs [81] and a commercially available magnetic liposome, Metafectene ${ }^{\circledR}$ Pro [82], were shown to display three sequential modes of intracellular dynamics following cell internalization.

Vercauteren and coworkers developed a novel dynamic colocalization algorithm based on dual-color particle tracking and trajectory correlation analysis [83]. Similar to the earlier findings with PEI/DNA NPs $[78,79]$, they demonstrated that a polymeric gene vector based on bioreducible poly(amido amine), $\mathrm{p}$ (CBA-ABOL)/DNA NPs, was contained in endolysosomal vesicles [83]. They showed that $\mathrm{p}(\mathrm{CBA}-\mathrm{ABOL}) / \mathrm{DNA}$ NPs were contained in Rab5-positive sorting endosomes, followed by LAMP1and Rab7-positive late endosomes/lysosomes. Most of the particles were trapped within vesicles up to $24 \mathrm{~h}$, highlighting the importance of vesicle escape as a barrier [83]. Akita and coworkers also used dual-color particle tracking to demonstrate that a liposome formulation previously reported to internalize into cells via macropinocytosis [84] underwent directional transport within endosomes in an MTdependent manner, but the velocity was slower than "free" AdV that escaped endosomes and endosomes devoid of the liposomes [76]. Interestingly, the liposome movement was highly restricted when MTs were disrupted by nocodazole, similar to what others observed with PEI/DNA NPs [6], whereas the diffusion of free AdV was relatively unhindered at the identical condition [76]. More recently, Kim and coworkers investigated the intracellular dynamics of a non-viral gene vector based on a copolymer of polylysine and polyethylene glycol $\left(\mathrm{CK}_{30} \mathrm{PEG}_{10 \mathrm{k}} / \mathrm{DNA} \mathrm{NPs}\right)$ [85] that, as previously described, has been tested in clinical trials for cystic fibrosis (CF) gene therapy [59]. They found that the $\mathrm{CK}_{30} \mathrm{PEG}_{10 \mathrm{k}} / \mathrm{DNA}$ NPs preferentially traffic through a caveolin-mediated pathway rather than an endo-lysosomal pathway, but were actively transported at a similar velocity to motor proteins carrying caveolin along the MT [85].

Together, these findings suggest that therapeutic NPs internalized by cells by various endocytic pathways can utilize MTs and motor proteins to rapidly approach the nucleus via active transport [85]. However, NP physiochemical properties can influence the mode of internalization, and therefore, the type and motility of the NP-containing vesicles. Lai and coworkers found that $24 \mathrm{~nm}$ carboxylate-modified polystyrene NPs entered live cells via a novel non-degradative, cholesterol independent, non-clathrin- and non-caveolae-mediated pathway [86]. In contrast, otherwise identical $43 \mathrm{~nm}$ NPs accumulated in lysosomes due to clathrin-mediated endocytosis [86]. Lai and coworkers then went on to investigate the intracellular dynamics of the NPs, and found that the non-degradative vesicles (containing $24 \mathrm{~nm}$ NPs) trafficked toward the nucleus at a 4-fold slower rate than the acidic late endosomes and lysosomes (containing $43 \mathrm{~nm} \mathrm{NPs}$ ); the reduction in average transport rate was mostly due to a 3-fold smaller fraction of nondegradative vesicles that were being actively transported [87]. The difference in trafficking further confirmed that the $24 \mathrm{~nm}$ NPs were taken up by a mechanism distinct from the $43 \mathrm{~nm}$ NPs. Regardless of the relatively lower fraction of actively transported vesicles containing $24 \mathrm{~nm}$ NPs, the non-degradative nature of the vesicles may lead to overall improved delivery of nucleic acids to the nucleus or nearby organelles [87].

\subsubsection{Strategies to improve cytoplasmic dynamics of therapeutic nanoparticles}

Although several scientists have shown that synthetic NPs can be actively transported in cellular vesicles toward the nucleus via MTs, particles that escape these vesicles or that are internalized via nonendocytic mechanisms must penetrate the molecularly crowded cytoplasm to reach the nucleus. Cytoskeletal elements and cellular organelles may pose steric and/or adhesive barriers to efficient cytoplasmic transport of NPs [88]. Indeed, many synthetic NPs for gene and drug delivery possess hydrophobic or charged surfaces, which facilitate particle interactions with cytoplasmic components. Suh and coworkers thus tested whether shielding the NP surface with PEG chains can reduce cytoplasmic particle adhesion and, hence, increase NP diffusion rates [89]. In order to isolate cytoplasmic transport from endosomal trafficking, they microinjected hydrophobic polystyrene (PS) NPs and PEG-coated PS (PS-PEG) into the cytoplasm of live cells. They found using particle tracking that PEGylation markedly increased the fraction of NPs undergoing unhindered diffusion in the cytoplasm, leading to a 2-fold increase in the average diffusion rates of PS-PEG compared to PS NPs [89].

Based on the lessons from previous studies, NPs bearing moieties that mimic motor proteins would presumably be actively transported in an MT-dependent manner. Akita and coworkers demonstrated that a majority of PEGylated liposomes displayed random motion, whereas PEGylated liposomes decorated with a dynein light chain (LC8)associated peptide (derived from an African swine fever virus protein p54) could use MT-dependent active transport without the need for a vesicular sorting system [90]. Likewise, the cytoplasmic transport rate of microinjected plasmid DNA was greatly increased in a directional manner when a sequence that specifically binds to MT-associated proteins was inserted into the promoter of the plasmid DNA [91]. Modulation of MTs may also improve MT-mediated transport of NPs. Based on the previous finding that a highly acetylated MT remained stable following pharmacological or mechanical MT disruption, Badding and coworkers investigated whether increasing the level of acetylation resulted in enhanced cytoplasmic transport of plasmid DNA [92]. They showed that plasmid DNA displayed greater net rates of movement and longer runs of continuous motion toward the nucleus on highly acetylated MTs compared to those with fewer modifications [92]. However, it is not clear how this approach could be used safely in vivo.

\subsubsection{Dynamics of therapeutic nanoparticles near the cell nucleus}

Albeit to a lesser extent, dynamics of NPs in the nuclear area, which generally refers to the region surrounding the nucleus, have also been explored using particle tracking. Seisenberger and coworkers demonstrated that AAV moved similarly in the nuclear area compared to cytoplasm despite being somewhat slower [7]. More than half of the tracked AAV exhibited normal diffusion, but a sizable fraction of AAV displayed unidirectional motions that could be prevented with nocodazole treatment, indicating MT-mediated active transport [7]. However, motor proteins such as kinesin or dynein, as well as MTs, have not been found within the nucleus. This finding can be rather explained by well-described continuous tubular structures between the nucleus and cytoplasm [93,94]. Suh and coworkers observed PEI/DNA NPs that appeared to be intranuclear, but were more likely to be within cytoplasmic invaginations that extend into the nucleus [6].

The nuclear import process of PEI/DNA NPs has been investigated in mitotic cells using particle tracking [79]. In this study, PEI/DNA NPs moved toward the spindle apparatus during mitosis, underscoring that nuclear import of PEI/DNA was largely dependent upon cell division [79]. It should be noted that most of the cells in the human body are not actively dividing, rendering the nuclear envelope among the most challenging barriers to nuclear delivery of therapeutic NPs. 


\subsubsection{Intracellular dynamics of therapeutic nanoparticles in $3 D$}

The aforementioned studies utilized 2D particle tracking techniques to understand the intracellular dynamics of various NPs. However, unlike certain extracellular milieus, such as the vitreous gel, intracellular environments are anisotropic on length scales relevant to NP transport, such that approximations for extrapolating 2D behavior to the third dimension may not provide accurate measurements of NP transport rates. To address this issue, several groups have used 3D particle tracking technology to investigate cellular uptake and cytoplasmic transport processes of gene vectors. Coppola and coworkers demonstrated that lipoplexes primarily underwent Brownian or directional motions [95], similar to the observations in 2D intracellular particle tracking studies [6]. They showed that the fraction of directionally transported lipoplexes was markedly reduced when the cells were pretreated with actin filament-disrupting latrunculin B or MT-disrupting nocodazole, underscoring cytoskeleton-dependent active transport of lipoplexes in cells [95]. Katayama and coworkers also investigated intracellular transport of DNA polyplexes in tubulin-eGFP expressing cells using 3D particle tracking combined with dual color widefield imaging [96]. The imaging allowed for visualization of the individual polyplexes and their interactions with the fluorescently labeled MTs. Using this technique, the authors confirmed the previous findings that lipoplexes travel along the MTs in a directional fashion [96]. More recently, Welsher and Yang investigated the early stages of cellular uptake, including NP approach and attachment to the cell membrane, and NP entering of the cells, by overlaying 3D trajectories of HIV-1 Tat modified NPs with live imaging of 3D cellular contours from two-photon laser scanning microscopy [5]. Similar to an earlier 2D study [7] (Fig. 6A), Welsher and Yang observed "kissand-run" events between the NP and the cell surface (Fig. 6B). However, tracking in 3D allowed for much longer observation of the NP transport dynamics than is achievable in the 2D plane; Welsher and Yang observed deceleration of a NP earlier than theoretically predicted as it approached the cell membrane, possibly due to interaction with cellular receptors. They also observed correlations between NP transport and nanoscale cell membrane features, including anisotropic motion along cell filipodia, which they attributed to association between the NP and actin protrusions (Fig. 6) [5]. Thus, the advent of 3D particle tracking technology has both validated the cellular particle dynamics observed using 2D methods, while providing new insights to NP uptake and trafficking in live cells.

\subsection{Broader biomedical applications of particle tracking analysis}

\subsubsection{Particle tracking as a method for probing microrheology}

The macroscopic viscoelasticity of biological fluids, such as that measured by a rheometer or viscometer, gives insight into the macroscopic behavior of that fluid, such as the shear-thinning properties of mucus secretions that provide lubrication. However, characterizing the microscopic rheological properties of complex biological fluids not only gives insight about the structure and function of the fluids themselves at the length scale of many cellular processes, but also insight into the nano- to micro-scale barrier properties of these biological fluids to NPs for drug and gene delivery.

Particle tracking microrheology (PTM) relates particle motion to the linear viscoelastic shear moduli of the surrounding fluid. It aims to measure the same macroscale rheology that would be obtained using a traditional bulk rheometer or viscometer, but it is applicable to systems with minute sample volumes where traditional rheometry is infeasible, and it is sensitive to microstructure and mesoscale spatial heterogeneity $[97,98]$. As discussed later in this section, it has also been used to probe the length-scale dependent rheology experienced by microscale particles diffusing through a complex environment [9]. The mathematics of microrheology, including how to calculate rheological parameters from particle tracking data, is discussed in detail elsewhere [97-99].

PTM was first employed to characterize complex fluids containing high molecular weight polyethylene oxide [99], DNA [97], and actin [100]. Later studies demonstrated the power of PTM for probing cellular microstructure, including the relatively increased stiffness of the interphase nucleus compared to the cytoplasm [101], the spatial uniformity of the viscoelasticity in the cytoplasm of developing Caenorhasbditis elegans embryos [102], the relationship between matrix stiffness and cellular stiffness [103], and the changes in mechanical properties of cancer cells in response to chemotherapy treatment [104]. In these cases, the drag exerted by the fluid on the NP reveals the local elasticity or viscosity.

More recently, PTM analysis has been extended to characterization of biological fluids that act as extracellular barriers to NP drug and gene delivery, such as mucus. In this case, NPs that do not interact with the network components of the mucus gel can provide information about the fluid contained within the pores, and the steric barrier properties (pore size) of the network. Lai and coworkers used PTM to
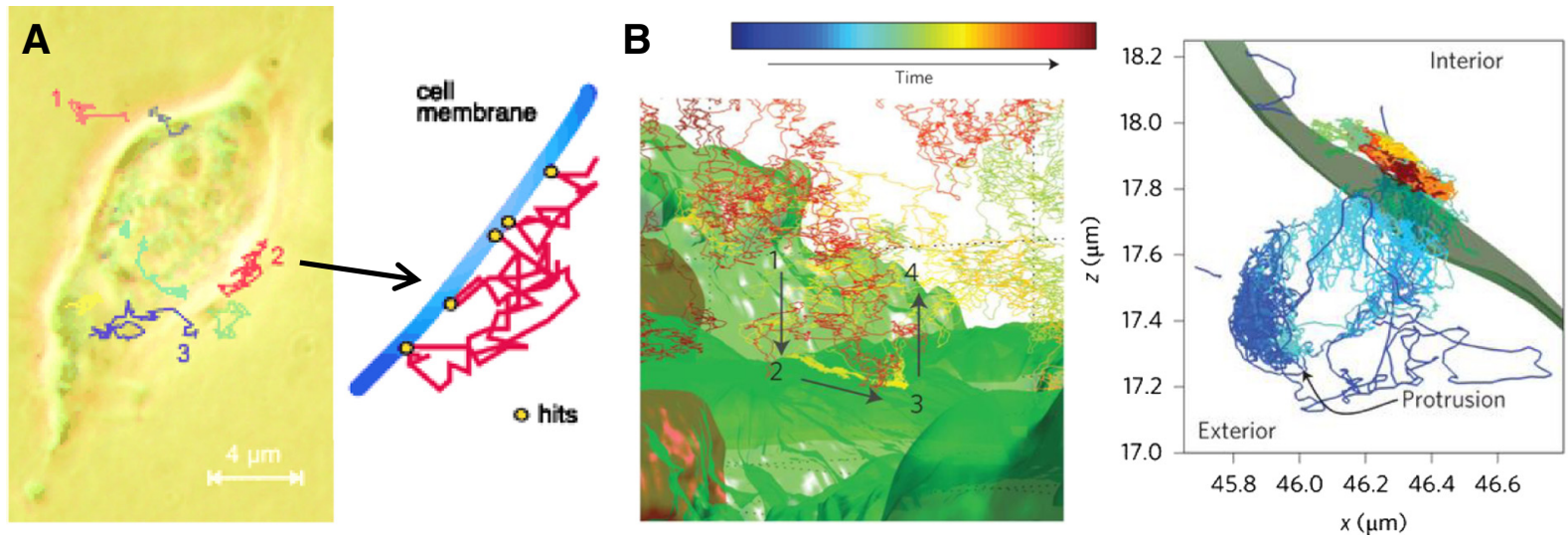

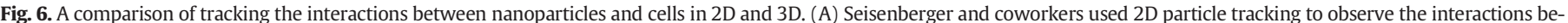

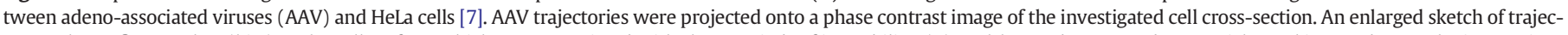

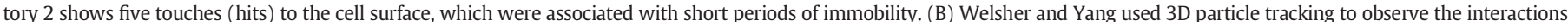

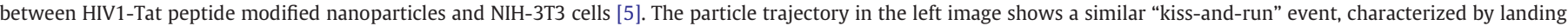

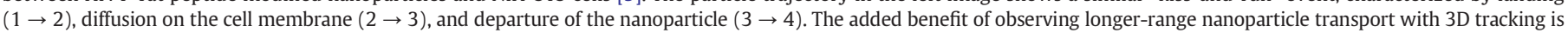
illustrated in the right image. The nanoparticle diffusion appears to make a 3D rendering of a protrusion from the cell membrane. Modified with permission from [5,7]. 
characterize the microrheological properties of fresh, undiluted human cervicovaginal mucus (CVM), as experienced by muco-inert, densely PEG-coated (PS-PEG) NPs ranging in size from 100 to $1000 \mathrm{~nm}$ [9]. The muco-inert PEG surface coating minimizes interactions between NPs and mucus, which is required for characterization of the local viscosity of the fluid within the pores of the mucus mesh, as well as the size of the pores. Particle tracking revealed that 100-500 nm PS-PEG NPs probed distances far larger than their sizes, and exhibited Brownian trajectories. In contrast, 1000 nm PS-PEG NPs exhibited constrained trajectories, indicative of a NP surrounded by a solid material that impeded its motion. Consequently, CVM posed a low elastic modulus ( $\left.\mathrm{G}^{\prime}\right)$ to 100 $500 \mathrm{~nm}$ PS-PEG NPs at all frequencies probed, whereas the $\mathrm{G}^{\prime}$ of CVM experienced by $1000 \mathrm{~nm}$ PS-PEG NPs was increased hundreds to thousands-fold at the same frequencies. They then investigated the effect of adding a non-ionic detergent commonly used in spermicides (non-oxynol 9, or "N9") on the CVM microrheology. They found that the $200 \mathrm{~nm}$ PS-PEG NPs experienced increased viscoelasticity in N9-treated CVM, likely indicating debundling of mucin strands that decreased the average pore size, thus increasing the steric restriction to diffusion of smaller NPs [9]. This type of structural information for CVM is of importance to NP-based drug delivery to the vagina, as the distribution of NPs experiencing a more elastic local environment would be restricted.

Bansil and coworkers used PTM to characterize the microrheology of mucin gels as a function of $\mathrm{pH}$ and in the presence of Helicobacter pylori [105]. Although the focus of the work was motility of H. pylori in the context of infection in the stomach, it highlighted the importance of mucus as a steric barrier to particles and pathogens, and the influence that infections and inflammation can have on mucus structure [105]. Such characterization is important, particularly in the context of disease, as changes in extracellular barrier structure influence the design criteria for an effective NP-based drug or gene delivery system. Georgiades and coworkers also used PTM to study the effect of pH on reconstituted porcine stomach mucin gels [106]. Although the relative effects of different reducing and cross-linking agents on the reconstituted gel structure can be elucidated, it is likely that the absolute measures of mucus viscoelasticity will change when using native mucus containing lipids, other proteins, cells, and various other components that are not present in purified mucin gels.

\subsubsection{Intravital particle tracking}

Intravital microscopy techniques allow for imaging inside the living body in real time. Intravital particle tracking has been used to indirectly observe blood flow velocity with tracer particles in the systemic circulation [107-110] and in the colonic microcirculation in experimental inflammatory bowel disease (IBD) [111], as well as to indirectly observe decreases in cerebrospinal fluid flow after traumatic brain injury [112]. More recently, intravital particle tracking techniques have been adapted to tracking NPs for drug and gene delivery in vivo in real time. As discussed previously in Section 2.2.2, Nance, Woodworth, and coworkers characterized the diffusion of densely PEG-coated (PS-PEG) and carboxylate-modified PS (PS-COOH) NPs in ex vivo rat and human brain tissue [2]. They also used intravital particle tracking to demonstrate that similar NP transport was observed in the live mouse brain in vivo: PS-PEG NPs up to $114 \mathrm{~nm}$ in size could freely diffuse in the brain ECM, while PS-PEG NPs $\sim 200 \mathrm{~nm}$ in size were sterically hindered (Fig. 2A). PS-COOH NPs of all sizes were adhesively immobilized in brain tissue (Fig. 2A). As another example of the correlation between the trajectories of NPs, in this case observed by particle tracking ex vivo and in vivo, and in vivo distribution over physiologically relevant distances, they showed that only NPs that diffused in brain tissue (69 and $114 \mathrm{~nm}$ PS-PEG NP) were able to distribute widely throughout the brain after intracranial injection (Fig. 2B). This technique highlighted the importance of NP surface properties for achieving widespread distribution within the brain upon direct NP application to the brain, as when using convection enhanced delivery (CED), or as might be expected after NP extravasation from the bloodstream due, for example, to a leaky BBB in tumors. It also further emphasized the importance of using non-adhesive NPs in characterizing the structure of biological barriers, as prior estimates of the brain ECM pore size using adhesive NPs indicated that NPs as large as $\sim 114 \mathrm{~nm}$ in size would not penetrate through brain tissue [2].

Intravital microscopy may also be used to visualize NPs at various stages of the delivery process in vivo. Using particle tracking in live mice to track the movement of anti-HER2 antibody-conjugated quantum dots (Qdots) in a subcutaneous HER2-overexpressing breast cancer tumor, Tada and coworkers visualized Qdots initially in circulation, and as they extravasated into the tumor, moved within the tumor extracellular matrix (ECM), bound to the cell membrane, and became internalized [113]. The average speed of the Qdots in circulation was 100 to $600 \mu \mathrm{m} / \mathrm{s}$, but reduced to $1-4 \mu \mathrm{m} / \mathrm{s}$ prior to extravasation. Once in the tumor interstitium ( $\sim 2 \mathrm{~h}$ after IV injection), the Qdots diffused at an average rate $\left(0.0014 \mu \mathrm{m}^{2} / \mathrm{s}\right)$ that was $\sim 10,000$-fold slowed compared to free diffusion in solution $\left(\sim 10 \mu \mathrm{m}^{2} / \mathrm{s}\right)$. Within $6 \mathrm{~h}$ of injection, binding of Qdots to cell membranes in the tumor was observed; Qdot motion became restricted to a small area. Once intracellular, active transport of the Qdots toward the perinuclear region was observed [113]. In later work by the same group, Kawai and coworkers used this intravital particle tracking model to investigate the delivery of IV injected NPs as a function of size [114]. Qdots (20 nm) and fluorescent PS NPs (40, 100, and $200 \mathrm{~nm}$ ) were used. The time to extravasation and internalization in the tumor increased with increasing NP size, and $200 \mathrm{~nm}$ NPs did not extravasate. For the 20-100 nm NPs, the NP speed decreased at each stage as the path became more tortuous and restrictive, from perivascular localization to the tumor intercellular spaces. In addition, the speed at each stage tended to decrease as NP size increased [114].

\section{Implementing particle tracking in nanomedicine research: a quick-start guide}

Thanks to advances in hardware and free and open-source software, particle tracking can now be readily implemented by newcomers and is poised for broad use in the drug and gene delivery community. To facilitate the adoption and implementation of particle tracking, this section reviews state-of-the-art experimental designs, tracking algorithms, and data analysis techniques. We also discuss potential pitfalls that researchers may encounter when conducting particle tracking experiments and analyzing the resulting data, and we suggest strategies to overcome these challenges.

\subsection{Design of particle tracking experiments: particles, microscope, and camera}

Time-lapse videos of particles in a specimen are the raw data in particle tracking experiments. Collecting videos with the highest possible quality - in short, bright particles with high contrast against a uniform background - simplifies data analysis and enables reliable interpretation. Here we address three critical factors that influence video quality: the particles, microscope, and camera. Optical properties of the biological specimen under investigation also impact the quality of videos that can be acquired, but those factors are typically beyond the researcher's control.

A variety of particles and illumination schemes have been used for particle tracking, including micron-sized colloids imaged via brightfield illumination [13] and gold NPs via darkfield microscopy [115]. However, we focus our review on fluorescent particles and fluorescence microscopy, which are particularly useful for identifying drug and gene delivery NPs in heterogeneous biological materials. Particles that are intensely fluorescent compared to the sample autofluorescence can be more reliably identified and localized. Internal labeling of particles is often preferable compared to external labeling, as it is less likely to change the surface properties of the particle and alter the particle's interaction 
with the specimens of interest. Particles can be internally labeled by physically encapsulating the fluorescent dye in the particle core (though this strategy is discouraged if dye leaks from the particle), or by chemically conjugating the dye to a polymer from which particles are then formulated $[2,3]$. Many fluorescent dyes, with various chemical reactivities, are commercially available, though photostable dyes with high quantum yield and extinction coefficient should be selected whenever possible [116]. Alternatively, bright, photostable, internally-labeled fluorescent polystyrene nanospheres are commercially available, and they are often used as model drug and gene carrier NPs in particle tracking studies $[1,47,48,52,64]$. The color of the fluorophore is another critical consideration. Biological specimens often have high autofluorescence, especially at shorter excitation wavelengths, which can interfere with particle detection and tracking. Red or dark red fluorescent dyes may be advantageous [57,117], if a light source and a detection scheme optimized for such dyes are available.

Although some research groups use microscopes specially designed and optimized for particle tracking, custom-built instrumentation usually is not required. In fact, particle tracking videos can be collected using hardware common in biomedical research labs and core facilities [118]. For tracking of fluorescent particles, a widefield microscope with epifluorescence illumination and appropriate fluorescence filter sets is typically used. Objectives with large numerical aperture are ideal for high resolution particle tracking, since they collect more light, and tracking precision is proportional to the square root of the number of photons emitted by a fluorescent particle [118]. A high-powered light source, such as a mercury or metal halide arc lamp, should be used at its highest intensity setting. Then, the exposure time of the camera should be adjusted so that the image is neither under- nor over-exposed [118]. This procedure will minimize particle tracking errors, as discussed in Section 4.2.4. The microscope should be placed on a pneumatic vibration-isolation table to dampen any external vibrations, such as from footsteps and lab equipment. Depending on the experiment, it may be necessary to maintain the specimen at physiological temperature. If the microscope stage is to be heated for the experiment, the temperature should be at equilibrium before collecting tracking videos, to prevent sample drift arising from expansion or contraction of microscope components.

A sensitive (high quantum efficiency), low-noise camera is essential for precise particle tracking. The best choice is an electron-multiplying charge coupled device (EMCCD) camera, though scientific complementary metal oxide semiconductor (sCMOS) cameras can be used as well [119]. The required frame rate will depend on the particle dynamics and research question. We have found 15 frames per second to be of sufficient frequency for studying transport of particles with diameters on the order of $100 \mathrm{~nm}$ in biological fluids and tissues, including mucus [48,64], vitreous [28], and brain tissue [2]. Faster frame rates will be necessary for studying more rapid movement [118]. If the exposure time can be set independently of the frame rate, the exposure should be no longer than necessary to achieve bright particle images. The rationale behind this guideline will be discussed in the section on errors in particle tracking. Although particle tracking videos are large files, it is important that the data be saved in a "lossless" file format that does not compress the file by discarding information [118].

Two rules should be kept in mind when preparing specimens for particle tracking experiments. First, when possible, the chamber containing the specimen should be well sealed to minimize sample evaporation and convective flow during the experiment $[64,120]$. Second, the particle concentration must be appropriate for visualizing and tracking individual particles. The average particle spacing must be larger than the average particle displacement from frame to frame, or else the paths of multiple particles will intermingle, making it difficult to accurately construct individual trajectories [13]. If the particles are moving very rapidly, a faster frame rate can be used, but if that is not sufficient or feasible, a new specimen with lower particle concentration should be prepared. Particle concentration should also be kept low (volume fraction $<0.01$ ) so that interactions between particles are unimportant compared to thermal forces [121]. To minimize dilution when adding particles to the specimen, simply prepare a more concentrated particle stock and add a small volume of it to the specimen. Specimen dilution of a few percent by volume, or less, can be readily achieved.

When performing particle tracking using unfamiliar hardware or software, it is helpful to track particles in a simple fluid with known viscosity, like water, and verify that the diffusion coefficient can be measured to within a few percent of the value predicted by the StokesEinstein equation [13]. Note that simple systems give cleaner images than most biological systems; treat them as a best-case scenario.

\subsection{Extracting quantitative measurements from particle tracking videos: automated particle tracking using the Crocker and Grier algorithm}

There are two main stages to automated particle tracking: 1) locating the spatial coordinates of particles in each frame of the movie, and 2) linking the coordinates through time into probable trajectories. Typically, tracking algorithms only require a video of particles and a few parameters, such as the apparent particle size and the maximum frame-to-frame particle displacement, as their inputs. As output, tracking algorithms provide the particle trajectories, from which the mean squared displacement (MSD) can be calculated. We focus our review on the tracking algorithm of Crocker and Grier [13]. Theirs and similar algorithms [12] have been widely used in fields such as colloid science, microrheology, biophysics, and, more recently, drug and gene delivery. The Crocker and Grier algorithm was originally implemented in the IDL programming language, but it has since been translated into various other languages, including Python, MATLAB, and C ++ [122]. Numerous alternative algorithms have been developed for tracking particles, which we briefly discuss later.

In the algorithm of Crocker and Grier, particle coordinates are identified in four steps: 1) use common image-processing techniques to prepare the image; 2) find local maxima of brightness that may correspond to particles; 3 ) hone in on each candidate particle's exact center with subpixel precision; and 4) discern which of the candidates are true particles based on their morphology and measured brightness. A paper [13] and an online tutorial [123] explain these steps in precise mathematical and practical detail, respectively. Here, we will conceptually describe each step in intermediate detail, sufficient to allow ready adoption by the drug and gene delivery community.

The software first prepares the images by applying a spatial bandpass filter to remove features with small-scale variation (e.g., camera noise) and large-scale variation (e.g., uneven lighting). This effectively erases objects that are much smaller or much larger than the size of the particles. Fig. 7 demonstrates how background noise and autofluorescence in a CF sputum sample (Fig. 7A) can be removed with a spatial bandpass filter to isolate only the brightly fluorescent NP (Fig. 7B). Next, the software identifies all local maxima in the processed image to provide an initial estimate of where particles may be found. If two or more local maxima are separated by a distance smaller than the particle diameter, the software only retains the brightest one. Then, the software iterates to identify the intensity-weighted centroid ("center of mass") of each spot - that is, the average position of the pixels in the spot, weighted by brightness. This step makes it possible to resolve the location of the particle center to a precision much better than 1 pixel. Finally, the software characterizes each spot by its total brightness, size, and eccentricity (deviation from circular shape); true particles tend to appear bright and circular. Using these attributes, spots that correspond to particles can be distinguished, and the rest discarded, with minimal user input.

At this point, the software has identified the locations of particles in each image (frame). Next, the locations must be linked together across frames into trajectories. The algorithm robustly handles the complications of real trajectories: Particles are allowed to leave the frame, new particles are allowed to enter, and particles can even be tracked if they temporarily vanish and reappear nearby within a user-specified 
A

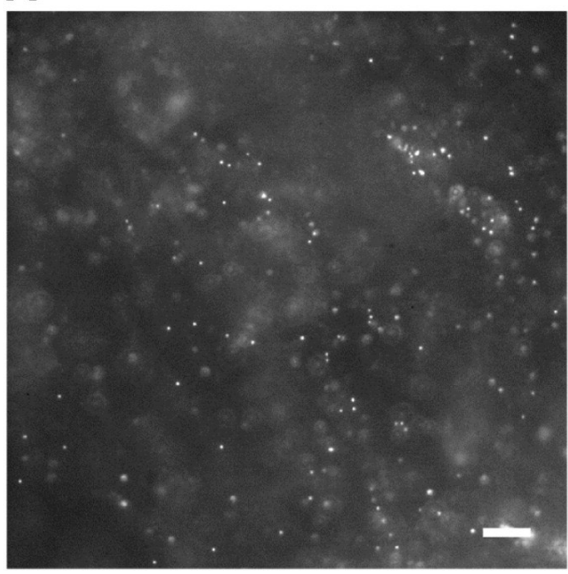

B

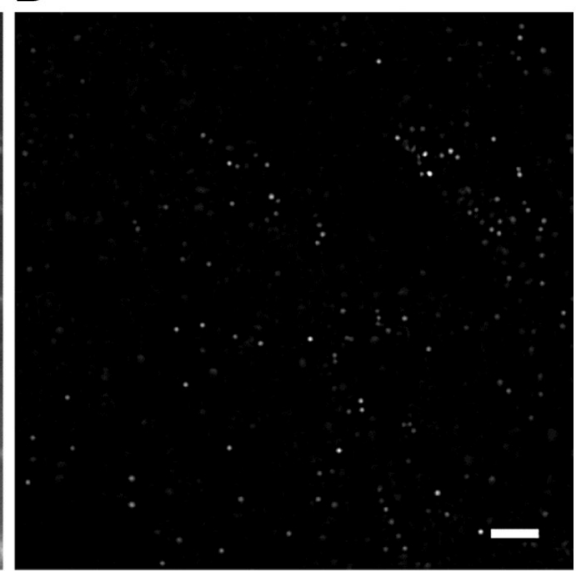

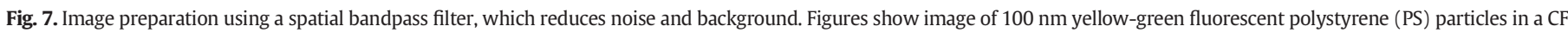
sputum sample (A) before and (B) after processing with a bandpass filter. Scale bars represent $10 \mu \mathrm{m}$.

number of frames. If particles typically do not move far between frames relative to their separation, the task of linking them is simple and unambiguous. If there are many quickly moving particles in the field of view, the Crocker and Grier linking algorithm links particle locations between frames in a way that minimizes the total length of the links. This procedure is grounded in the statistics of random walks; a diffusing particle is most likely to be found near where it was last seen. The linking algorithm is rigorously correct for non-interacting particles undergoing Brownian diffusion [13], and it is employed effectively in a variety of tracking applications. (See Section 4.3.2 for more on trajectory linking.)

As the concentration of particles increases, trajectory linking becomes more difficult. To simplify the problem, the algorithm requires that the user specify the maximum particle displacement allowed from one frame to the next. The simplest option for selecting the maximum displacement is to watch the particles move through several frames and estimate the upper range of their movement between frames. For Brownian motion, the Stokes-Einstein equation can also be used to estimate a reasonable maximum particle displacement. After trajectory linking is complete, the user can inspect a histogram of displacements at the shortest time scale. If the histogram decays to zero as the displacement approaches the maximum displacement, then the choice of maximum displacement was appropriate. However, if a substantial fraction of particles moved the specified maximum displacement between frames, then the trajectory linking should be redone with a larger maximum displacement. If, on the other hand, few particle displacements are nearly as large as the specified maximum displacement, then the maximum displacement may be decreased for better performance and accuracy.

To process a set of videos with automated tracking, only a few minutes of user attention are required to input parameters and ensure that the specified parameters are suitable. A standard computer can typically process at least one video frame per second, though this depends on the number of particles (and therefore the number of calculations needed for a single frame) in the video, as well as the exact automated tracking algorithm and implementation used. Thus, the software can process a typical video of a few hundred frames in a few minutes. The code will output the trajectories in a text file, spreadsheet, or database. Subsequent data analysis will depend on the scientific question under investigation.

\subsection{Basic analysis}

Broadly, analysis of particle tracking data consists of reducing a large number of particle positions over time to a physically descriptive number or a simple distribution. For drug and gene delivery applications, we are generally concerned with whether particles can diffuse through biological barriers and reach target sites. Particle tracking can also can reveal information about the rheology and microstructure of the medium itself. In either case, the analysis begins by using particle trajectories to calculate the displacement of the particles over time.

A simple random-walk trajectory can be summarized by its mean squared displacement (MSD) : $\left\langle\Delta \mathrm{r}^{2}(\mathrm{t})\right\rangle=\left\langle\left[\mathbf{r}\left(\mathrm{t}^{\prime}+\mathrm{t}\right)-\mathbf{r}\left(\mathrm{t}^{\prime}\right)\right]^{2}\right\rangle$, where $\mathbf{r}$ is the particle position, the brackets indicate an average over starting times $\mathrm{t}^{\prime}=0$ to $\mathrm{t}^{\prime}=\mathrm{T}-\mathrm{t}, \mathrm{T}$ is the duration of the trajectory, and $\mathrm{t}$ is lag time. The lag time is a time interval, not a clock time. Our earlier review [14] provides a detailed explanation of lag time, referred to therein generically as "time scale." Briefly, the displacement of a particle between any two positions along its trajectory can be calculated, and the time elapsed between the appearance of the particle at those two positions is the lag time. For a single particle, all the squared displacements with lag time $t$ are averaged to give $\left\langle\Delta r^{2}(t)\right\rangle$. The longer a trajectory is observed, the more observations $\mathbf{r}\left(\mathrm{t}^{\prime}+\mathrm{t}\right)-\mathbf{r}\left(\mathrm{t}^{\prime}\right)$ contribute to the MSD over each lag time interval t. To further improve the statistics, the MSDs of many particles may be averaged into an ensemble MSD, $\left\langle\left\langle\Delta \mathrm{r}^{2}(\mathrm{t})\right\rangle\right\rangle$, where the double brackets indicate averaging both over time and over multiple particles.

Then, $\left\langle\left\langle\Delta \mathrm{r}^{2}(\mathrm{t})\right\rangle\right\rangle$ is interpreted using a physical model. In a simple viscous fluid, the MSD is linear and proportional to the diffusion coefficient, D: In two dimensions, $\left\langle\left\langle\Delta \mathrm{r}^{2}(\mathrm{t})\right\rangle\right\rangle=4 \mathrm{Dt}$. Alternatively, the MSD may grow slower than linearly, indicating subdiffusive motion. Or the MSD may grow faster than linearly, indicating superdiffusive motion associated with active systems or a combination of directed (ballistic) motion and diffusion. Saxton and Jacobson [124] and others [14,124, 125] provide simple primers on these physical interpretations and their associated functional forms.

A common complication arises from vibration and drift (Table 1), in which the portion of the sample in view vibrates with the surrounding environment or flows convectively due to pressure gradients. This ballistic motion alters the trajectories and MSD, and it is usually undesirable. Drift can be mitigated by tightly sealing the sample chamber and allowing sufficient time for the sample to equilibrate before collecting particle tracking videos. However, in many experimental scenarios, drift or vibration may be impossible to avoid. If the drift is uniform over the field of view and many particles are present $(\mathrm{N} \gg 10)$, their collective motion can be subtracted away [118]. Many implementations of the Crocker and Grier algorithm include an option for drift subtraction. This "correction" inevitably introduces some error [118], but it should be performed whenever drifting motion is apparent across the sample. 
Table 1

Sources of error in particle tracking, and how to minimize them.

\begin{tabular}{|c|c|c|c|}
\hline & Source of error & How to minimize & How to determine magnitude of problem \\
\hline Static error & Noise reduces localization precision & $\begin{array}{l}\text { - Use bright particles } \\
\text { - Use sensitive, low-noise camera }\end{array}$ & Track particles affixed to coverslip and measure MSD \\
\hline Dynamic error & Particles move during exposure time & Use short exposure time & $\begin{array}{l}\text { Examine images for particles that appear blurry and } \\
\text { asymmetric }\end{array}$ \\
\hline Drift & $\begin{array}{l}\text { Field of view moves due to convection } \\
\text { or shaking }\end{array}$ & $\begin{array}{l}\text { - Isolate sample from air currents } \\
\text { - Calculate average motion of all particles and subtract } \\
\text { that from the trajectories }\end{array}$ & $\begin{array}{l}\text { Examine trajectories for directed motion or motions that } \\
\text { appear synchronized across the ensemble }\end{array}$ \\
\hline
\end{tabular}

\section{Advanced topics in particle tracking}

\subsection{Data analysis and interpretation in "messy," heterogeneous biological environments}

A key advantage of particle tracking is its ability to capture and characterize heterogeneity in particle transport behavior. This is valuable in certain drug and gene delivery applications, where only a fraction of particles may be required to reach the target to have a therapeutic effect. In contrast, techniques that only provide ensemble averages may miss small but critical subpopulations of particles. Particle tracking can similarly reveal heterogeneity within a complex biological material. For example, the trajectory map of individual $100 \mathrm{~nm}$ NPs in water reveals relatively uniform, diffusive behavior (Fig. 8A). In comparison, even with their non-adhesive coatings, $100 \mathrm{~nm}$ PS-PEG NPs experience different local microenvironments in CF sputum, as illustrated by the heterogeneity in the particle trajectories (Fig. 8B). Since the displacements of NPs in water follow a normal distribution, the individual particle MSDs (Fig. 9A) can be averaged, and a single diffusion coefficient can represent the entire ensemble. In a more complex, heterogeneous system like CF sputum, the individual particle MSDs can vary significantly (Fig. 9B), and there is not necessarily a single value that can describe the entire ensemble $[57,126,127]$.

In some systems $[126,128]$ the MSDs of individual particles are well separated into two or more modes, such as a group of diffusive particles and a group of immobilized particles with MSDs that quickly asymptote to a fixed value. Once the groups are identified, the particles within each group can be averaged. In other systems, the variation between individual particle MSDs is less well separated. Valentine and coworkers developed a formal approach for distinguishing when trajectories are statistically distinct [129]. Particles can be systematically partitioned, and each distinct environment can be individually characterized.
If particles experiencing different microenvironments are averaged together, the overall result can be distorted. Highly mobile particles leave the field of view more quickly, and thus the MSD at long lag times is weighted by slow particles [118]. On the other hand, if one anomalously mobile trajectory is averaged with a collection of less mobile ones, it will greatly distort the mean [130]. We recommend that any anomalous trajectories be treated as part of a separate ensemble. Moreover, if heterogeneity is an important aspect of the system, the individual MSDs need not be averaged at all.

In order to achieve adequate statistical power when calculating an ensemble-averaged MSD, the number of tracked particles and the duration of the trajectories must be carefully considered. A trajectory with $\mathrm{N}$ time points contains $\mathrm{N}-\mathrm{t}$ intervals of length $\mathrm{t}$. That is, $\mathrm{N}-\mathrm{t}$ total displacements can be temporally averaged to obtain the MSD over lag time t. However, since the intervals overlap, the measurements are not strictly independent. As a general rule, these $\mathrm{N}-\mathrm{t}$ intervals comprise $1.5 \mathrm{~N} / \mathrm{t}$ statistically independent measurements [129]. The rule was arrived at empirically; other approaches have also been published [131]. In concrete terms, an MSD data point with $<10 \%$ error requires 100 statistically independent measurements [118]. For example, to obtain a single-particle MSD for lag times out to $t=15$ frames, the particle would need to be observed for $\mathrm{N}=1000$ frames. However, if the MSDs for P particles can be averaged, N can be reduced to $1000 / \mathrm{P}$ frames. As an example of adequate statistical power, Nance and coworkers calculated the ensemble average MSD of NPs diffusing in brain tumor tissue at $\mathrm{t}=15$ frames for $\mathrm{P}>500$ particle trajectories, each of which were $\mathrm{N} \geq 15$ frames long [30].

Particle tracking in samples from patients, for instance, may reveal substantial sample-to-sample variation. This challenge is not unique to particle tracking, but rather is common to many measurements of complex biological samples. Increasing the sample size may reveal patterns such as correlations between disease state and particle tracking results.
A

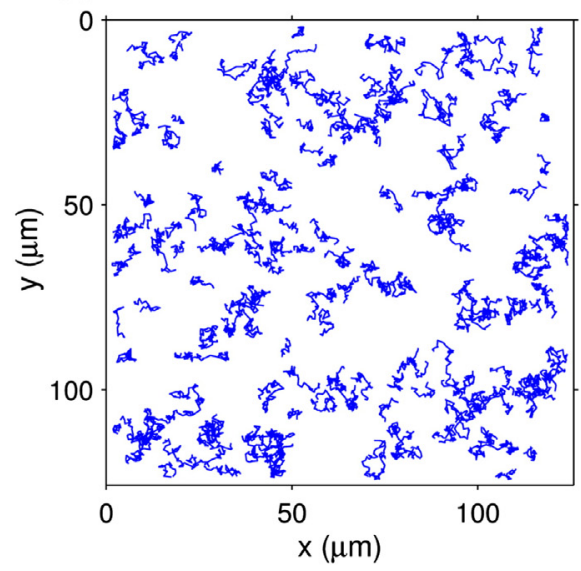

B

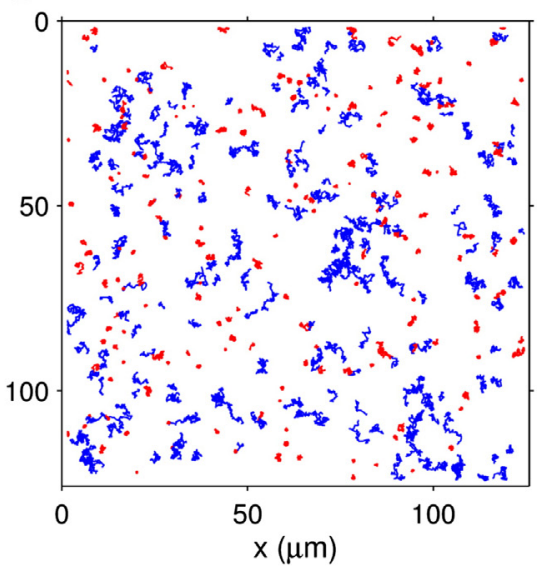

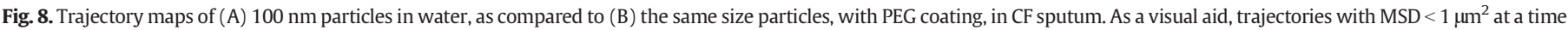

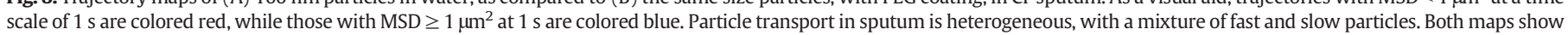
300 frames of motion, collected at 20 frames per second. (For interpretation of the references to color in this figure legend, the reader is referred to the web version of this article.) 


\section{A}

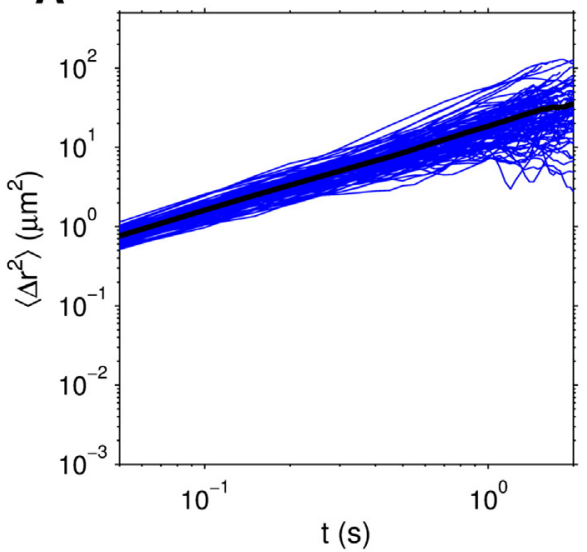

B

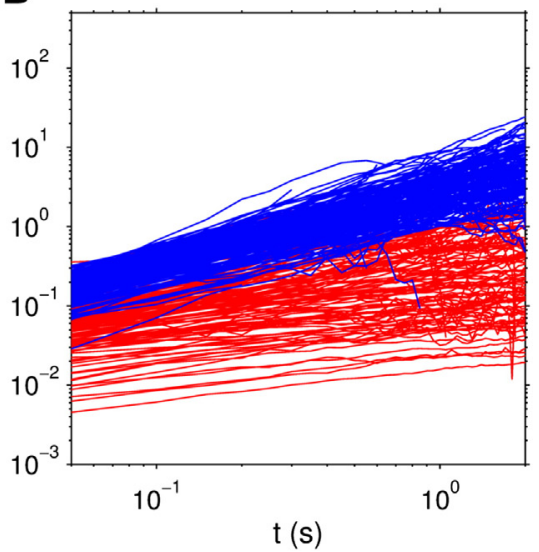

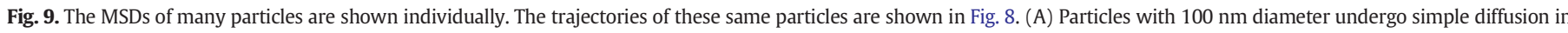

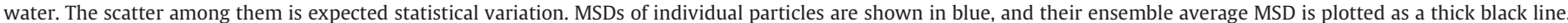

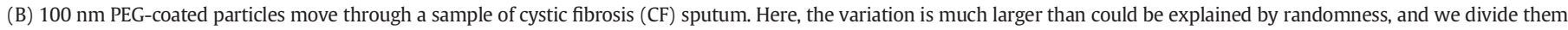

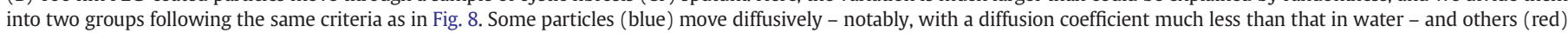

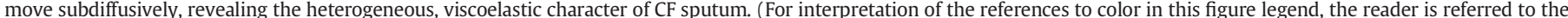
web version of this article.)

New computational strategies to help manage large data sets from high-throughput particle tracking are discussed in Section 4.4.

\subsection{High-precision particle tracking and methods to minimize error}

Failure to recognize and account for imprecision and inaccuracy in particle tracking data can lead to misinterpretation [132,133]. Precision refers to uncertainty in location, and is defined as the standard deviation of the measured locations when a single stationary particle is imaged and localized multiple times. In contrast, accuracy describes whether the position estimates deviate from the true particle position with systematic bias [132,134]. Below, we discuss factors that impact accuracy and precision in particle tracking experiments and how to deal with them.

\subsubsection{Resolution in particle tracking}

Tracking resolution (i.e., localization precision) is different than microscope resolution. As classically defined, microscope resolution is the minimum distance between two distinguishable points. The resolution of a traditional light microscope is approximately $250 \mathrm{~nm}$, half the wavelength of visible light [135]. However, this limit does not apply to locating the center of one isolated object. Using an appropriate algorithm, it is possible to localize the center of an isolated particle or point source to better than the diffraction-limited resolution $[132,136]$. In fact, researchers can routinely track particles with subpixel resolution and achieve a lateral spatial precision of tens of nanometers or less using a standard light microscope [118]. If a particle's image spans multiple pixels - ideally, more than three pixels across - we can find its position with subpixel accuracy by taking the average position of these pixels, weighted by brightness, as discussed in Section 3.2. Alternative methods for calculating particle position with subpixel resolution are discussed in Section 4.3.1.

\subsubsection{Static error}

Tracking precision is fundamentally limited by photon noise: a particle emits photons stochastically, and consequently, there is statistical uncertainty in locating the particle from its image (Fig. 10 and Table 1) [137]. The uncertainty in particle position is inversely proportional to the square root of the number of detected photons $[118,137]$. Fig. 10 illustrates that bright particles (Fig. 10A-D) can be localized more precisely than dim particles (Fig. 10E-H). Additionally, experimental limitations related to detector and specimen properties can
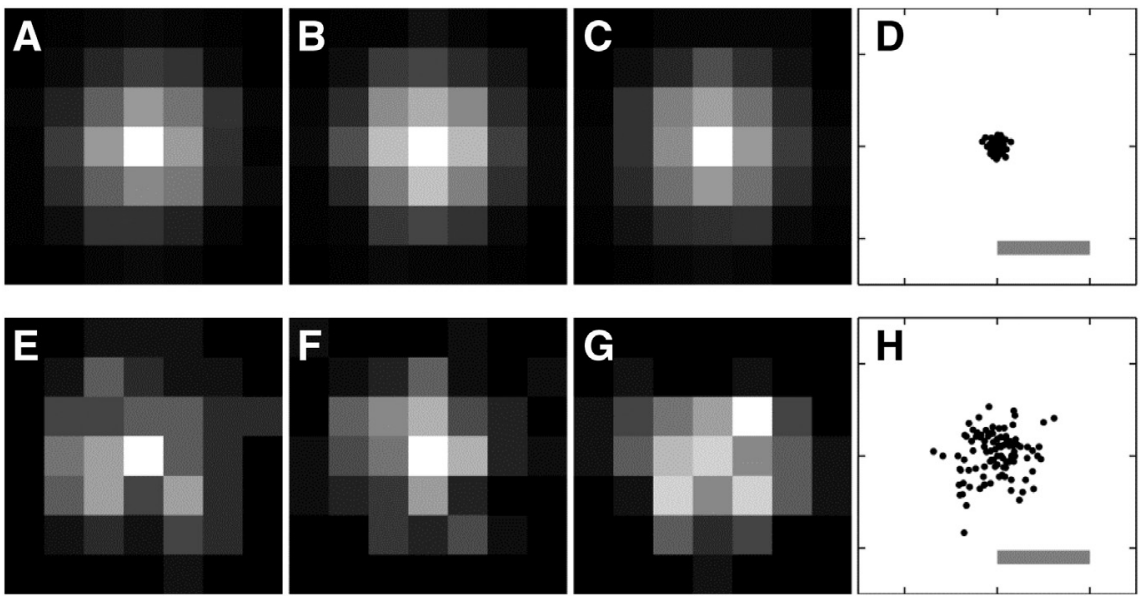

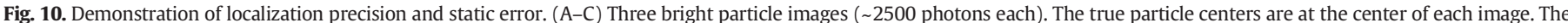

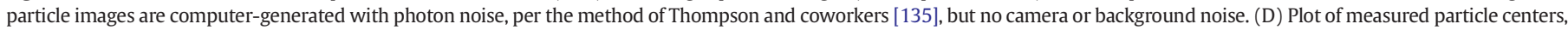

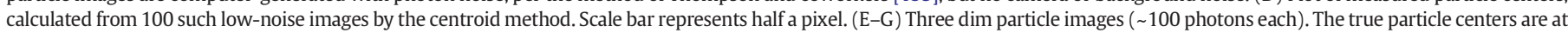
the center of each image. (H) Plot of measured particle centers, calculated from 100 such high-noise images by the centroid method. Scale bar represents half a pixel. 
further degrade precision in determining particle locations. Detector noise includes dark current (thermally-induced electrons in the detector) and readout noise (errors in reading the number of photoelectrons built up in a pixel) $[132,135]$. Detector noise is greatly reduced in high-performance, cooled, EMCCD cameras. Specimen autofluorescence and out-of-focus background fluorescence can also reduce localization precision and accuracy, which can be an issue when tracking particles in a thick biological specimen using standard epifluorescence illumination. Image processing can remove some, but not all, of this background [132]. Finally, lateral localization precision of fluorescent particles is best for particles located in the focal plane - where the particle image is smallest, brightest, and closest to a Gaussian curve in shape - and decreases with increasing distance from the focal plane [132,138].

This inability to exactly localize particles has been referred to as static error, localization error, or random error $[115,118,120]$. Static error adds a constant offset to the MSD, as derived previously [115]: $\mathrm{MSD}_{\text {measured }}=\mathrm{MSD}_{\text {true }}+2 \sigma^{2}$, where $\sigma$ is the localization uncertainty. A perfectly immobilized particle will have a true MSD $=0$ for all lag times, but because of static error, the actual measured MSD will be a non-zero constant, MSD $=2 \sigma^{2}[115,139]$. However, in most particle tracking experiments, the true underlying MSD is not known a priori, so it may not be immediately evident how to separate the measured MSD into the true component and artifact.

In addition to the random error associated with camera noise and pixelation, particle localization is subject to systematic errors, wherein particles can be "biased" toward certain locations, such as pixel centers. Several experimental details in both hardware and software are implicated in these errors [139]. Of course, if the particles typically move much more than a pixel between frames, optimizing subpixel resolution is less important. Readers with applications where particles frequently move in subpixel steps are encouraged to consult the referenced literature and consider these details with care.

\subsubsection{Dynamic error}

Additional error is introduced by motion blur. When a particle moves substantially during the camera exposure time, a blurred spot is recorded, representing the time integral of the particle's location (Table 1 and Fig. 11). Particle motion during the exposure time reduces the observed MSD at short times for randomly diffusing particles, since they tend to revisit previously explored regions $[118,140]$. This effect has been called dynamic error [120]. In addition, particle motion increases static error, because motion blur reduces localization precision. In fact, this can be important, as motion blur reduces localization precision by roughly two-fold under typical experimental conditions, as compared to stationary particles [141].

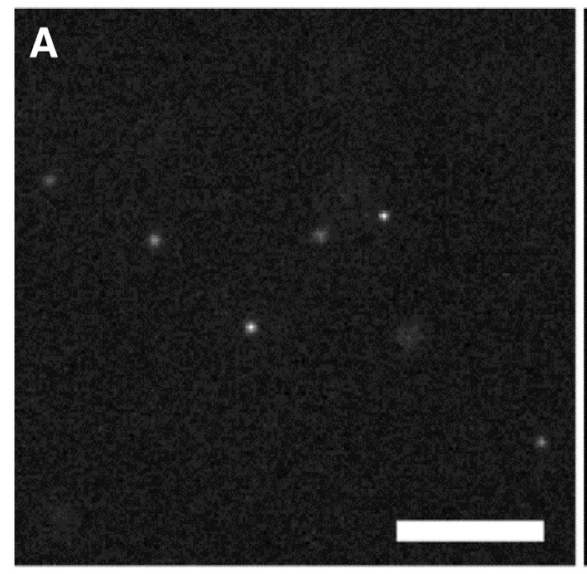

Like static error, dynamic error can alter the measured MSD, and can thus introduce inaccuracies when using the MSD to calculate diffusion coefficients and viscoelastic moduli. Static error alone adds a constant offset to the MSD, which flattens the MSD at short time scales on a log-log plot, and makes diffusive motion appear sub-diffusive [115]. Dynamic error, by itself, decreases the MSD at short time scales, which makes diffusive motion appear like active transport. Static and dynamic errors act in opposite directions, so depending on which type of error is larger, one effect may dominate, or occasionally, the two effects may largely cancel each other [120]. Both sources of error are more pronounced at short time scales, when the true MSD is smaller.

\subsubsection{Minimizing error}

It is critical to ensure that potential sources of error are minimized prior to collecting data, as it is difficult, if not impossible, to completely correct for error after the experiment. To boost signal-to-noise ratio and reduce static error, researchers should choose bright particles, a microscope objective with large numerical aperture, and a sensitive camera with low noise. The only way to reduce dynamic error is to use an exposure time that is small compared to the frame interval $[118,120]$. Unfortunately, using a shorter exposure time to minimize dynamic error can increase static error (Fig. 11A), and using a longer exposure time to minimize static error can increase dynamic error (Fig. 11B). A compromise is to use the longest exposure time possible without introducing large dynamic error. For Brownian motion, an exposure time no longer than one quarter the frame interval will result in $<10 \%$ dynamic error at the shortest time scale, and even smaller dynamic error at longer time scales [118].

Dynamic error is especially difficult to correct after an experiment, because it is time-dependent and also depends on the underlying type of motion, which is often unknown [120]. Static error is simpler to quantify and correct, since static error adds a constant offset to the MSD regardless of the nature of particle motion. Static error can be estimated by tracking particles fixed to a coverslip under signal-to-noise conditions similar to those of the experiment, and then subtracted from the ensemble MSD [115,118]. However, this simple approach may not precisely mimic the background noise in the experiment, nor the effect that motion blur has on static error. An alternative method has recently been proposed for measuring localization precision of moving particles, though this technique requires a custom microscope configuration [141]. Tracking particles in water or other fluids of known viscosity and comparing their MSD to theory may also help reveal types and magnitudes of error in a particular particle tracking experiment setup.

How much do researchers conducting particle tracking experiments need to worry about static and dynamic errors? The answer depends both on the system under investigation and on the desired analysis. If

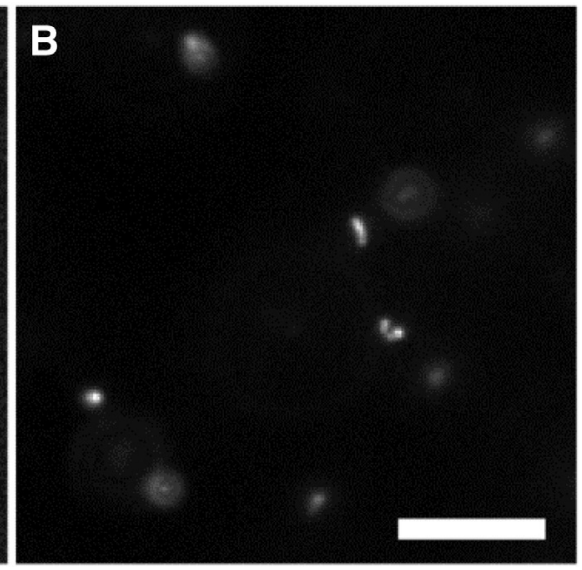

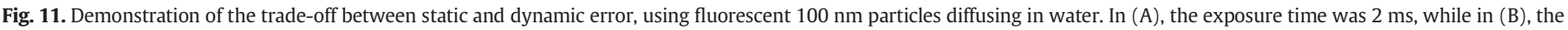

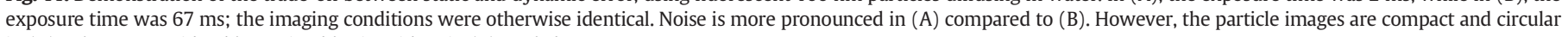
in (A), whereas considerable motion blur is evident in (B). Scale bars represent $10 \mu \mathrm{m}$. 
the research objective is to precisely quantify diffusion coefficients or viscoelastic moduli, or to fit a model to the data, it is critical to consider error to ensure proper analysis. In particular, higher spatial and temporal resolution are needed to accurately study particle motion at short time scales, where the MSD is smaller and the errors are proportionally larger. However, if the research objective is to qualitatively compare the transport of two types of NPs with very different behavior, such as mobile and immobile, moderate tracking errors require less thorough examination and are unlikely to alter the final conclusions.

\subsection{Enhanced algorithms for tracking in challenging biological environments}

Researchers conducting particle tracking experiments for drug and gene delivery applications must contend with imaging conditions that complicate particle localization and trajectory linking. The standard tracking methods described in Section 3.2 typically perform quite well, even in complex samples. However, newer methods may permit even more accurate characterization of particle transport behavior. We now outline select alternative approaches for particle localization and trajectory linking, and discuss their advantages and disadvantages. Regardless of the method chosen, proper algorithm implementation and careful choice of parameters are critical for minimizing error [142].

\subsubsection{Locating particles}

There are two main classes of algorithms for locating particle positions in two dimensions: those that use model fitting and those that do not. The model fitting methods find the particle location by varying model parameters such as the $\mathrm{x}$ and $\mathrm{y}$ spatial coordinates and, using a least squares method or maximum likelihood estimation (MLE), determine the mathematical model that best fits the particle image [119]. The MLE technique can, in theory, approach the best possible localization precision $[119,137]$. A point source that is in focus can be well-modeled by a Gaussian, though modeling asymmetric or out-offocus particles is not trivial [132-134]. Another limitation of fitting methods is that they tend to be computationally intensive and slow [133].

Particles can also be localized by methods that do not use model fitting. The centroid technique, described in Section 3.2, is perhaps the simplest and most widely used non-fitting method. Such algorithms may be well-suited for experimental conditions that distort the particle image, including rapid particle motion, imaging deep in tissue, and specimen heterogeneity [119], all of which are common in drug and gene delivery experiments. Indeed, although Gaussian fitting was reported to perform better than the centroid method at localizing immobilized particles, especially at low signal-to-noise ratios [132, 134 ], a recent study found that the centroid method is more reliable for mobile particles [141].

A newer non-fitting method locates particles using radial symmetry [133]. In essence, the radial symmetry method draws lines orthogonal to contours of constant intensity in the particle image. The point closest to the intersections of these lines is an estimate of the particle center. Radial symmetry was reported to perform better than the centroid method, and nearly as well as Gaussian fitting using MLE [133]. Radial symmetry was also 100 times faster than Gaussian fitting, though slower than the centroid method [133]. Development of new algorithms for localizing particles rapidly and precisely continues to be an active area of research, motivated recently by localization-based super-resolution microscopy techniques such as PALM/STORM, which are based on finding the centers of isolated fluorescent spots [143-145].

\subsubsection{Linking features into trajectories}

For most drug and gene delivery applications, particle tracking experiments can be designed such that the Crocker-Grier algorithm described in Section 3.2 is sufficient for trajectory linking. However, in certain cases, such as crowded intracellular environments, newer trajectory linking methods can provide more accurate results $[146,147]$. The Crocker-Grier algorithm does not account for trajectory merging or splitting. Merging may result from overlap of two particles in view, colloidal aggregation, or fusion. The Crocker and Grier algorithm would record the disappearance of one of the particles, and if the particle later "reappeared" (the trajectories split), it would be tracked separately from its earlier history. Thus, potential information about the particle's trajectory over longer time scales would be lost. A second, related shortcoming is that the algorithm considers only two frames at a time. In the jargon of algorithms, it is greedy: it finds the best trajectory linking through each individual time step, which may not coincide with the optimal global solution when the full trajectories are considered.

An alternative method by Jaqaman and coworkers [146] performs the usual frame-by-frame linking, but then revisits the full trajectories to reconcile gaps, splits, and merges in a globally optimized way. It evaluates the likelihood of a merge (overlap) and a split using both the proximity and appearance of the particles involved. For example, if one trajectory appears to end in the close vicinity of another trajectory, and that trajectory at once becomes brighter, a merge is probable. Rather than relying on the user-specified maximum possible particle displacement as a fixed search range, Jaqaman and coworkers use an adaptive search range based on local particle density and the prior trajectory of each particle; this produced marginally more accurate results, with increased computational efficiency [146].

In the techniques discussed thus far, all particles are located first, and then trajectories are linked. New, advanced methods that perform linking and localization together have been developed for contexts in the cell biology field where discerning features is especially challenging, with low signal-to-noise or high density. It is likely that such methods may be of value to the drug and gene delivery community in the future, as well. In the algorithm of Sergé and coworkers, linking informs the localization procedure, using the historical record of a particle's appearance and motion to guide where particles should be sought in the next frame [147]. Sergé and coworkers also take an innovative approach to identifying all potential particles in a crowded image. Since the apparent center of an individual particle can be distorted by bright neighboring particles, the brightest particles are identified first. Then, they are subtracted from the image, and identification is repeated to detect dimmer particles until only noise remains [147].

\subsection{Advanced microscopy techniques for particle tracking in thick specimens}

Many exciting innovations in light microscopy in recent years have been harnessed for improved particle tracking, including microscope configurations that reduce background fluorescence [148] and technologies that enable tracking in 3D [149,150]. Although these techniques often require custom instrumentation, some are already commercially available.

Light sheet fluorescence microscopy, also called selective plane illumination microscopy, has been attracting increasing interest recently as a technique to achieve reduced background fluorescence when imaging deep into thick biological specimens [151]. This technique requires two objective lenses oriented orthogonally to each other. One objective is used to form a thin light sheet, which illuminates only a narrow plane of the specimen, and thus background fluorescence is reduced. The other objective, perpendicular to the light sheet, is used for detection. Light sheet microscopy can thus achieve optical sectioning, similar to confocal microscopy, but with less photobleaching and faster acquisition rates. A number of recent papers report improved signal-to-noise ratio, and good spatial and temporal resolution, using light sheet microscopy-based particle tracking $[148,152,153]$.

Typically, particles are only tracked in 2D ( $\mathrm{x}$ and $\mathrm{y})$, but a range of approaches now exist for also localizing particles in the axial (z) dimension to obtain 3D trajectories [132,154]. One approach is to 
collect z-stacks with confocal microscopy, but even with a spinning disk confocal microscope, the temporal resolution is insufficient for tracking fast moving particles. A second approach is to track a single particle at a time, keeping it in focus by automatically adjusting the height of the stage or the objective lens as the particle moves [154-156]. A third approach exploits changes in the appearance of the particle as it goes in and out of focus; fluorescent particles in focus appear as compact Gaussian spots, whereas diffraction rings appear when the particle lies more than a few hundred nanometers from the focal plane. Even with a standard microscope, the diffraction rings may be used to approximate the depth of the particle $[13,138]$. To obtain better axial location precision, a number of microscope configurations have been invented that vary the particle's appearance in a predictable way as the particle moves in the $\mathrm{z}$ direction. Such configurations contain additional optical elements, such as a cylindrical lens, in the light path [149,157,158]. Alternatively, the microscope can be configured to image multiple planes of the specimen at once, from which the particle position in the $\mathrm{z}$ dimension can be calculated [150,159,160].

As mentioned at the beginning of Section 2.2, one limitation of highresolution multiple particle tracking is that particles typically can only be tracked for a few micrometers before they diffuse away from the microscope's focal plane. The first two approaches for 3D tracking can enable particles to be tracked over longer times and distances. We also note that complementary microscopy techniques, such as fluorescence recovery after photobleaching (FRAP), may be useful in studying diffusion of drug delivery particles over longer times and distances. FRAP has recently been reviewed elsewhere [161].

\subsection{Computational tools for reproducible and high-throughput particle tracking}

As tools for storing, sharing, and processing data become more widely available, more research is published with raw data and detailed information that enables others to reproduce key results from beginning to end with a reasonable investment of effort $[162,163]$. Open methods and well-documented, repeatable analysis procedures are increasingly common and sometimes required. Fortunately, several particle tracking software tools, including those discussed in detail above, are available as free, open source software. If researchers post their raw videos and their procedures, others in the community can study and verify their process with relative ease.

A growing concern is the sheer volume of data from particle tracking experiments. High-performance computing, which is becoming more available as well, can expedite the processing of large data sets. Massive, multi-core resources formerly restricted to large simulations-based research can be harnessed inexpensively by novices for small-batch use [164]. Particle location schemes process each frame independently, and so multiple frames can be processed simultaneously if parallel computing resources are available. Notably, consumer graphics processing units (GPUs) are naturally suited to image processing calculations, and could perform such analysis quickly and in parallel on a desktop computer. Use of GPUs for particle tracking analysis seems likely in the future.

\section{Outlook: future applications of particle tracking to drug and gene delivery research}

Nanoparticles (NPs) hold promise as efficacious drug and gene delivery systems. However, NPs encounter numerous barriers in the body prior to reaching their delivery targets. Particle tracking is a powerful technique for characterizing how effectively NPs overcome extracellular and cellular barriers, by allowing direct visualization and quantification of NP transport on an individual particle basis.

Future applications of particle tracking in drug and gene delivery research are vast. Looking ahead, we envision the implementation of high-throughput particle tracking studies to test libraries of NP formulations in biological samples of interest, such as blood, mucus, or tissue. Such a screening technique will allow for rapid identification of NP formulations that are likely to exhibit favorable in vivo delivery and warrant further development. In Section 2, we described how particle tracking has been employed to examine the effect of two critical design criteria, particle size and surface chemistry, on particle transport and fate in biological tissues and fluids. Particle shape is another important design criterion that can affect particle diffusion [165] and interaction with cells and tissues [166,167]. The effect of particle anisotropy on Brownian motion has been studied by tracking ellipsoidal particles in water [165]. The impact of particle shape on drug and gene delivery has not been extensively studied by particle tracking, but we anticipate that particle tracking can provide valuable insight here as well.

Intravital particle tracking, which is just starting to become possible thanks to advances in imaging techniques, is likely to have considerable impact on drug and gene delivery research. The ability to directly observe the journey of a NP from the initial injection, into the bloodstream, to the transport behavior inside target tissues and cells, will likely provide invaluable insight for improved NP design. For pulmonary delivery, in vivo investigations of mucociliary clearance and its effect on particle transport will be valuable for researchers designing nanomedicines for airway diseases. New technologies, such as micro-optical coherence tomography $(\mu \mathrm{OCT})$, are enabling high resolution in vivo imaging of the lung airways $[168,169]$. In oral delivery applications, it is still debated how and to what extent particles traffic from the digesta into GI tract cells or the systemic circulation [35]. New endoscopic imaging, including $\mu \mathrm{OCT}$, could be useful here as well. Advancements in particle tracking imaging techniques will also help to elucidate how particles can avoid clearance by the reticuloendothelial system, transport through the lymphatic system, traverse the blood-brain barrier, and accumulate in tumors.

\section{Acknowledgments}

This work was supported by the National Institutes of Health grants P01HL51811 (J.H.), R01CA164789 (J.H.), U19AI133127 (J.H., L.M.E.), and R01HD062844 (J.H., L.M.E.); the Cystic Fibrosis Foundation grant HANES07XX0 (J.H., J.S.S.); and the W.W. Smith Charitable Trust grant A1302 (L.M.E.). The authors thank Dr. Robert Leheny, and National Science Foundation grant CBET-1033985 (R.L.), for support and comments on the manuscript. We acknowledge Mia Kanzawa, Joshua Kays, and Katharina Maisel for their helpful discussions.

The mucus-penetrating particle technology described in this publication is being developed by Kala Pharmaceuticals. Justin Hanes is a co-founder of Kala. He owns company stock, which is subject to certain restrictions under Johns Hopkins University policy. The terms of this arrangement are being managed by Johns Hopkins University in accordance with its conflict of interest policies.

\section{References}

[1] S.K. Lai, D.E. O'Hanlon, S. Harrold, S.T. Man, Y.Y. Wang, R. Cone, J. Hanes, Rapid transport of large polymeric nanoparticles in fresh undiluted human mucus, Proc. Natl. Acad. Sci. U. S. A. 104 (2007) 1482-1487.

[2] E.A. Nance, G.F. Woodworth, K.A. Sailor, T.Y. Shih, Q. Xu, G. Swaminathan, D. Xiang, C. Eberhart, J. Hanes, A dense poly(ethylene glycol) coating improves penetration of large polymeric nanoparticles within brain tissue, Sci. Transl. Med. 4 (2012) 149ra19.

[3] L.M. Ensign, B.C. Tang, Y.Y. Wang, T.A. Tse, T. Hoen, R. Cone, J. Hanes, Mucuspenetrating nanoparticles for vaginal drug delivery protect against herpes simplex virus, Sci. Transl. Med. 4 (2012) 138ra79.

[4] A.J. Kim, N.J. Boylan, J.S. Suk, M. Hwangbo, T. Yu, B.S. Schuster, L. Cebotaru, W.G. Lesniak, J.S. Oh, P. Adstamongkonkul, A.Y. Choi, R.M. Kannan, J. Hanes, Use of single-site-functionalized PEG dendrons to prepare gene vectors that penetrate human mucus barriers, Angew. Chem. Int. Ed. 52 (2013) 3985-3988.

[5] K. Welsher, H. Yang, Multi-resolution 3D visualization of the early stages of cellular uptake of peptide-coated nanoparticles, Nat. Nanotechnol. 9 (2014) 198-203.

[6] J. Suh, D. Wirtz, J. Hanes, Efficient active transport of gene nanocarriers to the cell nucleus, Proc. Natl. Acad. Sci. U. S. A. 100 (2003) 3878-3882. 
[7] G. Seisenberger, M.U. Ried, T. Endress, H. Buning, M. Hallek, C. Brauchle, Real-time single-molecule imaging of the infection pathway of an adeno-associated virus, Science 294 (2001) 1929-1932.

[8] M. Breunig, S. Bauer, A. Goepferich, Polymers and nanoparticles: intelligent tools for intracellular targeting? Eur. J. Pharm. Biopharm. 68 (2008) 112-128.

[9] S.K. Lai, Y.Y. Wang, R. Cone, D. Wirtz, J. Hanes, Altering mucus rheology to “solidify" human mucus at the nanoscale, PLoS One 4 (2009) e4294.

[10] K. Braeckmans, K. Buyens, W. Bouquet, C. Vervaet, P. Joye, F. De Vos, L. Plawinski, L. Doeuvre, E. Angles-Cano, N.N. Sanders, J. Demeester, S.C. De Smedt, Sizing nanomatter in biological fluids by fluorescence single particle tracking, Nano Lett. 10 (2010) 4435-4442

[11] J. Gelles, B.J. Schnapp, M.P. Sheetz, Tracking kinesin-driven movements with nanometre-scale precision, Nature 331 (1988) 450-453.

[12] R.N. Ghosh, W.W. Webb, Automated detection and tracking of individual and clustered cell surface low density lipoprotein receptor molecules, Biophys. J. 66 (1994) 1301-1318.

[13] J.C. Crocker, D.G. Grier, Methods of digital video microscopy for colloidal studies, J. Colloid Interface Sci. 179 (1996) 298-310.

[14] J. Suh, M. Dawson, J. Hanes, Real-time multiple-particle tracking: applications to drug and gene delivery, Adv. Drug Deliv. Rev. 57 (2005) 63-78.

[15] A. Bootz, V. Vogel, D. Schubert, J. Kreuter, Comparison of scanning electron microscopy, dynamic light scattering and analytical ultracentrifugation for the sizing of poly(butyl cyanoacrylate) nanoparticles, Eur. J. Pharm. Biopharm. 57 (2004) 369-375.

[16] P.H. Brown, P. Schuck, Macromolecular size-and-shape distributions by sedimentation velocity analytical ultracentrifugation, Biophys. J. 90 (2006) 4651-4661.

[17] W. Fraunhofer, G. Winter, C. Coester, Asymmetrical flow field-flow fractionation and multiangle light scattering for analysis of gelatin nanoparticle drug carrier systems, Anal. Chem. 76 (2004) 1909-1920.

[18] B.J. Frisken, Revisiting the method of cumulants for the analysis of dynamic lightscattering data, Appl. Opt. 40 (2001) 4087-4091.

[19] W. Jiskoot, D.J.A. Crommelin, Methods for Structural Analysis of Protein Pharmaceuticals, AAPS Press, Arlington, VA, 2005.

[20] D. Walczyk, F.B. Bombelli, M.P. Monopoli, I. Lynch, K.A. Dawson, What the cell "sees" in bionanoscience, J. Am. Chem. Soc. 132 (2010) 5761-5768.

[21] M.P. Monopoli, C. Aberg, A. Salvati, K.A. Dawson, Biomolecular coronas provide the biological identity of nanosized materials, Nat. Nanotechnol. 7 (2012) 779-786.

[22] M.P. Monopoli, D. Walczyk, A. Campbell, G. Elia, I. Lynch, F.B. Bombelli, K.A. Dawson, Physical-chemical aspects of protein corona: relevance to in vitro and in vivo biological impacts of nanoparticles, J. Am. Chem. Soc. 133 (2011) 2525-2534.

[23] M. Lundqvist, J. Stigler, G. Elia, I. Lynch, T. Cedervall, K.A. Dawson, Nanoparticle size and surface properties determine the protein corona with possible implications for biological impacts, Proc. Natl. Acad. Sci. U. S. A. 105 (2008) 14265-14270.

[24] B. Naeye, H. Deschout, V. Caveliers, B. Descamps, K. Braeckmans, C. Vanhove, J. Demeester, T. Lahoutte, S.C. De Smedt, K. Raemdonck, In vivo disassembly of IV administered siRNA matrix nanoparticles at the renal filtration barrier, Biomaterials 34 (2013) 2350-2358.

[25] B. Naeye, K. Raemdonck, J. Demeester, S.C. De Smedt, Interactions of siRNA loaded dextran nanogel with blood cells, J. Control. Release 148 (2010) e90-e91.

[26] H. Ragelle, R. Riva, G. Vandermeulen, B. Naeye, V. Pourcelle, C.S. Le Duff, C. D'Haese, B. Nysten, K. Braeckmans, S.C. De Smedt, C. Jerome, V. Preat, Chitosan nanoparticles for siRNA delivery: optimizing formulation to increase stability and efficiency, J. Control. Release 176 (2014) 54-63.

[27] G.R. Dakwar, E. Zagato, J. Delanghe, S. Hobel, A. Aigner, H. Denys, K. Braeckmans, W. Ceelen, S.C. De Smedt, K. Remaut, Colloidal stability of nano-sized particles in the peritoneal fluid: towards optimizing drug delivery systems for intraperitoneal therapy, Acta Biomater. 10 (2014) 2965-2975.

[28] Q. Xu, N.J. Boylan, J.S. Suk, Y.Y. Wang, E.A. Nance, J.C. Yang, P.J. McDonnell, R.A. Cone, E.J. Duh, J. Hanes, Nanoparticle diffusion in, and microrheology of, the bovine vitreous ex vivo, J. Control. Release 167 (2013) 76-84.

[29] T.F. Martens, D. Vercauteren, K. Forier, H. Deschout, K. Remaut, R. Paesen, M. Ameloot, J.F. Engbersen, J. Demeester, S.C. De Smedt, K. Braeckmans, Measuring the intravitreal mobility of nanomedicines with single-particle tracking microscopy, Nanomedicine (London) 8 (2013) 1955-1968.

[30] E. Nance, C. Zhang, T.Y. Shih, Q. Xu, B.S. Schuster, J. Hanes, Brain-penetrating nanoparticles improve paclitaxel efficacy in malignant glioma following local administration, ACS Nano 8 (10) (2014) 10655-10664

[31] R.G. Thorne, C. Nicholson, In vivo diffusion analysis with quantum dots and dextrans predicts the width of brain extracellular space, Proc. Natl. Acad. Sci. U. S. A. 103 (2006) 5567-5572.

[32] C.S. Schneider, J.G. Perez, E. Cheng, C. Zhang, P. Mastorakos, J. Hanes, J.A. Winkles, G.F. Woodworth, A.J. Kim, Minimizing the non-specific binding of nanoparticles to the brain enables active targeting of Fn14-positive glioblastoma cells, Biomaterials 42 (2015) 42-51.

[33] P. Mastorakos, C. Zhang, S. Berry, Y. Oh, S. Lee, C.G. Eberhart, G.F. Woodworth, J.S Suk, J. Hanes, Highly PEGylated DNA nanoparticles provide uniform and widespread gene transfer in the brain, Adv. Healthcare Mater. (2015), http://dx. doi.org/10.1002/adhm.201400800.

[34] S.K. Lai, Y.Y. Wang, J. Hanes, Mucus-penetrating nanoparticles for drug and gene delivery to mucosal tissues, Adv. Drug Deliv. Rev. 61 (2009) 158-171.

[35] L.M. Ensign, R. Cone, J. Hanes, Oral drug delivery with polymeric nanoparticles: the gastrointestinal mucus barriers, Adv. Drug Deliv. Rev. 64 (2012) 557-570.

[36] M. Dawson, E. Krauland, D. Wirtz, J. Hanes, Transport of polymeric nanoparticle gene carriers in gastric mucus, Biotechnol. Prog. 20 (2004) 851-857.

[37] O. Lieleg, I. Vladescu, K. Ribbeck, Characterization of particle translocation through mucin hydrogels, Biophys. J. 98 (2010) 1782-1789.
[38] Y.Y. Wang, S.K. Lai, J.S. Suk, A. Pace, R. Cone, J. Hanes, Addressing the PEC mucoadhesivity paradox to engineer nanoparticles that "slip" through the human mucus barrier, Angew. Chem. Int. Ed. Engl. 47 (2008) 9726-9729.

[39] T. Yu, Y.Y. Wang, M. Yang, C. Schneider, W. Zhong, S. Pulicare, W.J. Choi, O. Mert, J. Fu, S.K. Lai, J. Hanes, Biodegradable mucus-penetrating nanoparticles composed of diblock copolymers of polyethylene glycol and poly(lactic-glycolic acid), Drug Deliv. Transl. Res. 2 (2012).

[40] M. Yang, S.K. Lai, Y.Y. Wang, W.X. Zhong, C. Happe, M. Zhang, J. Fu, J. Hanes, Biodegradable nanoparticles composed entirely of safe materials that rapidly penetrate human mucus, Angew. Chem. Int. Ed. 50 (2011) 2597-2600.

[41] B.C. Tang, M. Dawson, S.K. Lai, Y.Y. Wang, J.S. Suk, M. Yang, P. Zeitlin, M.P. Boyle, J. $\mathrm{Fu}$, J. Hanes, Biodegradable polymer nanoparticles that rapidly penetrate the human mucus barrier, Proc. Natl. Acad. Sci. U. S. A. 106 (2009) 19268-19273.

[42] L.M. Ensign, S.K. Lai, Y.Y. Wang, M. Yang, O. Mert, J. Hanes, R. Cone, Pretreatment of human cervicovaginal mucus with Pluronic F127 enhances nanoparticle penetration without compromising mucus barrier properties to herpes simplex virus, Biomacromolecules 15 (12) (2014) 4403-4409.

[43] S.K. Lai, Y.Y. Wang, K. Hida, R. Cone, J. Hanes, Nanoparticles reveal that human cervicovaginal mucus is riddled with pores larger than viruses, Proc. Natl. Acad. Sci. U. S. A. 107 (2010) 598-603.

[44] A. Macierzanka, A.R. Mackie, B.H. Bajka, N.M. Rigby, F. Nau, D. Dupont, Transport of particles in intestinal mucus under simulated infant and adult physiological conditions: impact of mucus structure and extracellular DNA, PLoS One 9 (2014) e95274.

[45] L.M. Ensign, A. Henning, C.S. Schneider, K. Maisel, Y.Y. Wang, M.D. Porosoff, R. Cone, J. Hanes, Ex vivo characterization of particle transport in mucus secretions coating freshly excised mucosal tissues, Mol. Pharm. 10 (2013) 2176-2182.

[46] M.E.V. Johansson, M. Phillipson, J. Petersson, A. Velcich, L. Holm, G.C. Hansson, The inner of the two Muc2 mucin-dependent mucus layers in colon is devoid of bacteria, Proc. Natl. Acad. Sci. U. S. A. 105 (2008) 15064-15069.

[47] N.N. Sanders, S.C. De Smedt, E. Van Rompaey, P. Simoens, F. De Baets, J. Demeester Cystic fibrosis sputum: a barrier to the transport of nanospheres, Am. J. Respir. Crit. Care Med. 162 (2000) 1905-1911.

[48] J.S. Suk, S.K. Lai, Y.Y. Wang, L.M. Ensign, P.L. Zeitlin, M.P. Boyle, J. Hanes, The penetration of fresh undiluted sputum expectorated by cystic fibrosis patients by non-adhesive polymer nanoparticles, Biomaterials 30 (2009) 2591-2597.

[49] M. Dawson, D. Wirtz, J. Hanes, Enhanced viscoelasticity of human cystic fibrotic sputum correlates with increasing microheterogeneity in particle transport, J. Biol. Chem. 278 (2003) 50393-50401.

[50] B. Amsden, An obstruction-scaling model for diffusion in homogeneous hydrogels, Macromolecules 32 (1999) 874-879.

[51] D.B. Hill, P.A. Vasquez, J. Mellnik, S.A. McKinley, A. Vose, F. Mu, A.G. Henderson, S.H Donaldson, N.E. Alexis, R.C. Boucher, M.G. Forest, A biophysical basis for mucus solids concentration as a candidate biomarker for airways disease, PLoS One 9 (2014).

[52] K. Forier, A.S. Messiaen, K. Raemdonck, H. Deschout, J. Rejman, F. De Baets, H. Nelis S.C. De Smedt, J. Demeester, T. Coenye, K. Braeckmans, Transport of nanoparticles in cystic fibrosis sputum and bacterial biofilms by single-particle tracking microscopy, Nanomedicine (London) 8 (2013) 935-949.

[53] P.K. Singh, A.L. Schaefer, M.R. Parsek, T.O. Moninger, M.J. Welsh, E.P. Greenberg, Quorum-sensing signals indicate that cystic fibrosis lungs are infected with bacterial biofilms, Nature 407 (2000) 762-764.

[54] A.S. Messiaen, K. Forier, H. Nelis, K. Braeckmans, T. Coenye, Transport of nanoparticles and tobramycin-loaded liposomes in Burkholderia cepacia complex biofilms, PLoS One 8 (2013).

[55] K. Hida, S.K. Lai, J.S. Suk, S.Y. Won, M.P. Boyle, J. Hanes, Common gene therapy vira vectors do not efficiently penetrate sputum from cystic fibrosis patients, PLoS One 6 (2011) e19919.

[56] U. Griesenbach, E.W.F.W. Alton, Gene transfer to the lung: lessons learned from more than 2 decades of CF gene therapy, Adv. Drug Deliv. Rev. 61 (2009) 128-139.

[57] B.S. Schuster, A.J. Kim, J.C. Kays, M.M. Kanzawa, W.B. Guggino, M.P. Boyle, S.M Rowe, N. Muzyczka, J.S. Suk, J. Hanes, Overcoming the cystic fibrosis sputum barrier to leading adeno-associated virus gene therapy vectors, Mol. Ther. 22 (2014) 1484-1493.

[58] J.S. Suk, N.J. Boylan, K. Trehan, B.C. Tang, C.S. Schneider, J.M.G. Lin, M.P. Boyle, P.L Zeitlin, S.K. Lai, M.J. Cooper, J. Hanes, N-acetylcysteine enhances cystic fibrosis sputum penetration and airway gene transfer by highly compacted DNA nanoparticles, Mol. Ther. 19 (2011) 1981-1989.

[59] M.W. Konstan, P.B. Davis, J.S. Wagener, K.A. Hilliard, R.C. Stern, L.J.H. Milgram, T.H. Kowalczyk, S.L. Hyatt, T.L. Fink, C.R. Gedeon, S.M. Oette, J.M. Payne, O. Muhammad, A.G. Ziady, R.C. Moen, M.J. Cooper, Compacted DNA nanoparticles administered to the nasal mucosa of cystic fibrosis subjects are safe and demonstrate partial to complete cystic fibrosis transmembrane regulator reconstitution, Hum. Gene Ther. 15 (2004) 1255-1269.

[60] N.J. Boylan, J.S. Suk, S.K. Lai, R. Jelinek, M.P. Boyle, M.J. Cooper, J. Hanes, Highly compacted DNA nanoparticles with low MW PEG coatings: in vitro, ex vivo and in vivo evaluation, J. Control. Release 157 (2012) 72-79.

[61] J.S. Suk, A.J. Kim, K. Trehan, C.S. Schneider, L. Cebotaru, O.M. Woodward, N.J. Boylan, M.P. Boyle, S.K. Lai, W.B. Guggino, J. Hanes, Lung gene therapy with highly compacted DNA nanoparticles that overcome the mucus barrier, J. Control. Release 178 (2014) 8-17.

[62] X.Y. Yang, K. Forier, L. Steukers, S. Van Vlierberghe, P. Dubruel, K. Braeckmans, S. Glorieux, H.J. Nauwynck, Immobilization of pseudorabies virus in porcine tracheal respiratory mucus revealed by single particle tracking, PLoS One 7 (2012).

[63] S.K. Lai, K. Hida, S. Shukair, Y.Y. Wang, A. Figueiredo, R. Cone, T.J. Hope, J. Hanes Human Immunodeficiency virus type 1 is trapped by acidic but not by neutralized human cervicovaginal mucus, J. Virol. 83 (2009) 11196-11200. 
[64] B.S. Schuster, J.S. Suk, G.F. Woodworth, J. Hanes, Nanoparticle diffusion in respiratory mucus from humans without lung disease, Biomaterials 34 (2013) 3439-3446.

[65] D. Putnam, Polymers for gene delivery across length scales, Nat. Mater. 5 (2006) 439-451.

[66] A. Schroeder, D.A. Heller, M.M. Winslow, J.E. Dahlman, G.W. Pratt, R. Langer, T. Jacks, D.G. Anderson, Treating metastatic cancer with nanotechnology, Nat. Rev. Cancer 12 (2012) 39-50.

[67] B. Sodeik, M.W. Ebersold, A. Helenius, Microtubule-mediated transport of incoming herpes simplex virus 1 capsids to the nucleus, J. Cell Biol. 136 (1997) 1007-1021.

[68] S. Sanlioglu, P.K. Benson, J. Yang, E.M. Atkinson, T. Reynolds, J.F. Engelhardt, Endocytosis and nuclear trafficking of adeno-associated virus type 2 are controlled by rac1 and phosphatidylinositol-3 kinase activation, J. Virol. 74 (2000) 9184-9196.

[69] M. Suomalainen, M.Y. Nakano, S. Keller, K. Boucke, R.P. Stidwill, U.F. Greber, Microtubule-dependent plus- and minus end-directed motilities are competing processes for nuclear targeting of adenovirus, J. Cell Biol. 144 (1999) 657-672.

[70] J.S. Bartlett, R. Wilcher, R.J. Samulski, Infectious entry pathway of adeno-associated virus and adeno-associated virus vectors, J. Virol. 74 (2000) 2777-2785.

[71] R. Kircheis, L. Wightman, E. Wagner, Design and gene delivery activity of modified polyethylenimines, Adv. Drug Deliv. Rev. 53 (2001) 341-358.

[72] D. Luo, W.M. Saltzman, Synthetic DNA delivery systems, Nat. Biotechnol. 18 (2000) 33-37.

[73] P.J. Xiao, R.J. Samulski, Cytoplasmic trafficking, endosomal escape, and perinuclear accumulation of adeno-associated virus type 2 particles are facilitated by microtubule network, J. Virol. 86 (2012) 10462-10473.

[74] J.S. Suk, J. Suh, S.K. Lai, J. Hanes, Quantifying the intracellular transport of viral and nonviral gene vectors in primary neurons, Exp. Biol. Med. (Maywood) 232 (2007) 461-469.

[75] O. Meier, U.F. Greber, Adenovirus endocytosis, J. Gene Med. 6 (Suppl. 1) (2004) S152-S163.

[76] H. Akita, K. Enoto, T. Masuda, H. Mizuguchi, T. Tani, H. Harashima, Particle tracking of intracellular trafficking of octaarginine-modified liposomes: a comparative study with adenovirus, Mol. Ther. 18 (2010) 955-964

[77] R.P. Kulkarni, K. Castelino, A. Majumdar, S.E. Fraser, Intracellular transport dynamics of endosomes containing DNA polyplexes along the microtubule network, Biophys. J. 90 (2006) L42-L44.

[78] J. Suh, Y. An, B.C. Tang, C. Dempsey, F. Huang, J. Hanes, Real-time gene delivery vector tracking in the endo-lysosomal pathway of live cells, Microsc. Res. Tech. 75 (2012) 691-697.

[79] R. Bausinger, K. von Gersdorff, K. Braeckmans, M. Ogris, E. Wagner, C. Brauchle, A. Zumbusch, The transport of nanosized gene carriers unraveled by live-cell imaging Angew. Chem. Int. Ed. Engl. 45 (2006) 1568-1572.

[80] S. Berezhna, S. Schaefer, R. Heintzmann, M. Jahnz, G. Boese, A. Deniz, P. Schwille New effects in polynucleotide release from cationic lipid carriers revealed by confocal imaging, fluorescence cross-correlation spectroscopy and single particle tracking, Biochim. Biophys. Acta 1669 (2005) 193-207.

[81] K. de Bruin, N. Ruthardt, K. von Gersdorff, R. Bausinger, E. Wagner, M. Ogris, C. Brauchle, Cellular dynamics of EGF receptor-targeted synthetic viruses, Mol Ther. 15 (2007) 1297-1305

[82] A.M. Sauer, K.G. de Bruin, N. Ruthardt, O. Mykhaylyk, C. Plank, C. Brauchle, Dynamics of magnetic lipoplexes studied by single particle tracking in living cells, J. Control. Release 137 (2009) 136-145.

[83] D. Vercauteren, H. Deschout, K. Remaut, J.F. Engbersen, A.T. Jones, J. Demeester, S.C. De Smedt, K. Braeckmans, Dynamic colocalization microscopy to characterize intracellular trafficking of nanomedicines, ACS Nano 5 (2011) 7874-7884.

[84] I.A. Khalil, K. Kogure, S. Futaki, S. Hama, H. Akita, M. Ueno, H. Kishida, M. Kudoh, Y. Mishina, K. Kataoka, M. Yamada, H. Harashima, Octaarginine-modified multifunctional envelope-type nanoparticles for gene delivery, Gene Ther. 14 (2007) 682-689.

[85] A.J. Kim, N.J. Boylan, J.S. Suk, S.K. Lai, J. Hanes, Non-degradative intracellular trafficking of highly compacted polymeric DNA nanoparticles, J. Control. Release 158 (2012) 102-107.

[86] S.K. Lai, K. Hida, S.T. Man, C. Chen, C. Machamer, T.A. Schroer, J. Hanes, Privileged delivery of polymer nanoparticles to the perinuclear region of live cells via a non-clathrin, non-degradative pathway, Biomaterials 28 (2007) 2876-2884.

[87] S.K. Lai, K. Hida, C. Chen, J. Hanes, Characterization of the intracellular dynamics of a non-degradative pathway accessed by polymer nanoparticles, J. Control. Release 125 (2008) 107-111.

[88] D. Lechardeur, A.S. Verkman, G.L. Lukacs, Intracellular routing of plasmid DNA during non-viral gene transfer, Adv. Drug Deliv. Rev. 57 (2005) 755-767.

[89] J. Suh, K.L. Choy, S.K. Lai, J.S. Suk, B.C. Tang, S. Prabhu, J. Hanes, PEGylation of nanoparticles improves their cytoplasmic transport, Int. J. Nanomedicine 2 (2007) 735-741.

[90] H. Akita, K. Enoto, H. Tanaka, H. Harashima, Particle tracking analysis for the intracellular trafficking of nanoparticles modified with African swine fever virus protein p54-derived peptide, Mol. Ther. 21 (2013) 309-317.

[91] M.A. Badding, E.E. Vaughan, D.A. Dean, Transcription factor plasmid binding modulates microtubule interactions and intracellular trafficking during gene transfer, Gene Ther. 19 (2012) 338-346.

[92] M.A. Badding, D.A. Dean, Highly acetylated tubulin permits enhanced interactions with and trafficking of plasmids along microtubules, Gene Ther. 20 (2013) 616-624.

[93] M. Fricker, M. Hollinshead, N. White, D. Vaux, Interphase nuclei of many mammalian cell types contain deep, dynamic, tubular membrane-bound invaginations of the nuclear envelope, J. Cell Biol. 136 (1997) 531-544.

[94] J.L. Broers, B.M. Machiels, G.J. van Eys, H.J. Kuijpers, E.M. Manders, R. van Driel, F.C. Ramaekers, Dynamics of the nuclear lamina as monitored by GFP-tagged A-type lamins, J. Cell Sci. 112 (Pt 20) (1999) 3463-3475.
[95] S. Coppola, F. Cardarelli, D. Pozzi, L.C. Estrada, M.A. Digman, E. Gratton, A. Bifone, C. Marianecci, G. Caracciolo, The role of cytoskeleton networks on lipid-mediated delivery of DNA, Ther. Deliv. 4 (2013) 191-202.

[96] Y. Katayama, O. Burkacky, M. Meyer, C. Brauchle, E. Gratton, D.C. Lamb, Real-time nanomicroscopy via three-dimensional single-particle tracking, ChemPhysChem 10 (2009) 2458-2464.

[97] T.G. Mason, K. Ganesan, J.H. vanZanten, D. Wirtz, S.C. Kuo, Particle tracking microrheology of complex fluids, Phys. Rev. Lett. 79 (1997) 3282-3285.

[98] T.M. Squires, T.G. Mason, Fluid mechanics of microrheology, Annu. Rev. Fluid Mech. 42 (2009) 413.

[99] T.G. Mason, D.A. Weitz, Optical measurements of frequency-dependent linear viscoelastic moduli of complex fluids, Phys. Rev. Lett. 74 (1995) 1250-1253.

[100] F. Gittes, B. Schnurr, P.D. Olmsted, F.C. MacKintosh, C.F. Schmidt, Microscopic viscoelasticity: shear moduli of soft materials determined from thermal fluctuations, Phys. Rev. Lett. 79 (1997) 3286-3289.

[101] Y. Tseng, J.S.H. Lee, T.P. Kole, I. Jiang, D. Wirtz, Micro-organization and visco-elasticity of the interphase nucleus revealed by particle nanotracking, J. Cell Sci. 117 (2004) 2159-2167.

[102] B.R. Daniels, B.C. Masi, D. Wirtz, Probing single-cell micromechanics in vivo: the microrheology of C. elegans developing embryos, Biophys. J. 90 (2006) 4712-4719.

[103] E.L. Baker, J. Lu, D.H. Yu, R.T. Bonnecaze, M.H. Zaman, Cancer cell stiffness: integrated roles of three-dimensional matrix stiffness and transforming potential, Biophys. J. 99 (2010) 2048-2057.

[104] A. El Kaffas, D. Bekah, M. Rui, J.C. Kumaradas, M.C. Kolios, Investigating longitudinal changes in the mechanical properties of MCF-7 cells exposed to paclitaxol using particle tracking microrheology, Phys. Med. Biol. 58 (2013) 923-936.

[105] R. Bansil, J.P. Celli, J.M. Hardcastle, B.S. Turner, The influence of mucus microstructure and rheology in Helicobacter pylori infection, Front. Immunol. 4 (2013) 310.

[106] P. Georgiades, P.D. Pudney, D.J. Thornton, T.A. Waigh, Particle tracking microrheology of purified gastrointestinal mucins, Biopolymers 101 (2014) 366-377.

[107] S.Y.Jung, S. Ahn, K.H. Nam, J.P. Lee, S.J. Lee, In vivo measurements of blood flow in a rat using X-ray imaging technique, Int. J. Cardiovasc. Imaging 28 (2012) 1853-1858.

[108] D.J. Ravnic, A. Tsuda, A. Turhan, J.P. Pratt, H.T. Huss, Y.Z. Zhang, S.J. Mentzer, Multiframe particle tracking in intravital imaging: defining Lagrangian coordinates in the microcirculation, Biotechniques 41 (2006) 597-601.

[109] D.J. Ravnic, Y.Z. Zhang, A. Tsuda, J.P. Pratt, H.T. Huss, S.J. Mentzer, Multi-image particle tracking velocimetry of the microcirculation using fluorescent nanoparticles, Microvasc. Res. 72 (2006) 27-33.

[110] V.N. Kilin, H. Anton, N. Anton, E. Steed, J. Vermot, T.F. Vandamme, Y. Mely, A.S. Klymchenko, Counterion-enhanced cyanine dye loading into lipid nano-droplets for single-particle tracking in zebrafish, Biomaterials 35 (2014) 4950-4957.

[111] A. Turhan, M.A. Konerding, A. Tsuda, D.J. Ravnic, D. Hanidziar, M. Lin, S.J. Mentzer, Bridging mucosal vessels associated with rhythmically oscillating blood flow in murine colitis, Anat. Rec. (Hoboken) 291 (2008) 74-82.

[112] G. Xiong, J.A. Elkind, S. Kundu, C.J. Smith, M.B. Antunes, E. Tamashiro, J.M. Kofonow, C.M. Mitala, S.C. Stein, M.S. Grady, E. Einhorn, N.A. Cohen, A.S. Cohen, Traumatic brain injury-induced ependymal ciliary loss decreases cerebral spinal fluid flow, J. Neurotrauma 31 (16) (2014) 1396-1404.

[113] H. Tada, H. Higuchi, T.M. Wanatabe, N. Ohuchi, In vivo real-time tracking of single quantum dots conjugated with monoclonal anti-HER2 antibody in tumors of mice, Cancer Res. 67 (2007) 1138-1144.

[114] M. Kawai, H. Higuchi, M. Takeda, Y. Kobayashi, N. Ohuchi, Dynamics of different-sized solid-state nanocrystals as tracers for a drug-delivery system in the interstitium of a human tumor xenograft, Breast Cancer Res. 11 (2009) R43.

[115] D.S. Martin, M.B. Forstner, J.A. Kas, Apparent subdiffusion inherent to single particle tracking, Biophys. J. 83 (2002) 2109-2117.

[116] D.B. Murphy, Fundamentals of Light Microscopy and Electronic Imaging, Wiley-Liss, New York, 2001

[117] M.J. Rust, M. Lakadamyali, B. Brandenburg, X. Zhuang, Single-virus tracking in live cells, Cold Spring Harb. Protoc. 2011 (2011)

[118] J.C. Crocker, B.D. Hoffman, Multiple-particle tracking and two-point microrheology in cells, Methods Cell Biol. 83 (2007) 141-178.

[119] A. Small, S. Stahlheber, Fluorophore localization algorithms for super-resolution microscopy, Nat. Methods 11 (2014) 267-279.

[120] T. Savin, P.S. Doyle, Static and dynamic errors in particle tracking microrheology, Biophys. J. 88 (2005) 623-638.

[121] P.C. Hiemenz, R. Rajagopalan, Principles of Colloid and Surface Chemistry, 3rd ed. Marcel Dekker, New York, 1997.

[122] D.B. Allan, T.A. Caswell, N.C. Keim, Trackpy - related projects, https://github. com/soft-matter/trackpy\#related-projects.

[123] J.C. Crocker, E.R. Weeks, Particle tracking using IDL, http://www.physics.emory. edu/faculty/weeks//idl/tracking.html.

[124] M.J. Saxton, K. Jacobson, Single-particle tracking: applications to membrane dynamics, Annu. Rev. Biophys. Biomol. Struct. 26 (1997) 373-399.

[125] F. Huang, C. Dempsey, D. Chona, J. Suh, Quantitative nanoparticle tracking: applications to nanomedicine, Nanomedicine (Lond.) 6 (2011) 693-700

[126] M.H. Lee, D.H. Reich, K.J. Stebe, R.L. Leheny, Combined passive and active microrheology study of protein-layer formation at an air-water interface, Langmuir 26 (2010) 2650-2658.

[127] A. Lubelski, I.M. Sokolov, J. Klafter, Nonergodicity mimics inhomogeneity in single particle tracking, Phys. Rev. Lett. 100 (2008) 250602.

[128] R.C. Spero, R.K. Sircar, R. Schubert, R.M. Taylor II, A.S. Wolberg, R. Superfine, Nanoparticle diffusion measures bulk clot permeability, Biophys. J. 101 (2011) 943-950.

[129] M.T. Valentine, P.D. Kaplan, D. Thota, J.C. Crocker, T. Gisler, R.K. Prud'homme, M. Beck, D.A. Weitz, Investigating the microenvironments of inhomogeneous soft 
materials with multiple particle tracking, Phys. Rev. E Stat. Nonlinear Soft Matter Phys. 64 (2001) 061506.

[130] J.R. Samaniuk, J. Vermant, Micro and macrorheology at fluid-fluid interfaces, Soft Matter 10 (2014) 7023-7033.

[131] H. Qian, M.P. Sheetz, E.L. Elson, Single particle tracking. Analysis of diffusion and flow in two-dimensional systems, Biophys. J. 60 (1991) 910-921.

[132] H. Deschout, F. Cella Zanacchi, M. Mlodzianoski, A. Diaspro, J. Bewersdorf, S.T. Hess, K. Braeckmans, Precisely and accurately localizing single emitters in fluorescence microscopy, Nat. Methods 11 (2014) 253-266.

[133] R. Parthasarathy, Rapid, accurate particle tracking by calculation of radial symmetry centers, Nat. Methods 9 (2012) 724-726.

[134] M.K. Cheezum, W.F. Walker, W.H. Guilford, Quantitative comparison of algorithms for tracking single fluorescent particles, Biophys. J. 81 (2001) 2378-2388.

[135] R.E. Thompson, D.R. Larson, W.W. Webb, Precise nanometer localization analysis for individual fluorescent probes, Biophys. J. 82 (2002) 2775-2783.

[136] N. Bobroff, Position measurement with a resolution and noise-limited instrument, Rev. Sci. Instrum. 57 (1986) 1152-1157.

[137] R.J. Ober, S. Ram, E.S. Ward, Localization accuracy in single-molecule microscopy, Biophys. J. 86 (2004) 1185-1200.

[138] T. Savin, P.T. Spicer, P.S. Doyle, A rational approach to noise discrimination in video microscopy particle tracking, Appl. Phys. Lett. 93 (2008).

[139] J.C. Crocker, M.T. Valentine, E.R. Weeks, T. Gisler, P.D. Kaplan, A.G. Yodh, D.A. Weitz, Two-point microrheology of inhomogeneous soft materials, Phys. Rev. Lett. 85 (2000) 888-891.

[140] H.C. Berg, Random Walks in Biology, Expanded ed. Princeton University Press, Princeton, NJ, 1993.

[141] H. Deschout, K. Neyts, K. Braeckmans, The influence of movement on the localization precision of sub-resolution particles in fluorescence microscopy, J. Biophotonics 5 (2012) 97-109.

[142] N. Chenouard, I. Smal, F. de Chaumont, M. Maska, I.F. Sbalzarini, Y. Gong, J. Cardinale, C. Carthel, S. Coraluppi, M. Winter, A.R. Cohen, W.J. Godinez, K. Rohr, Y. Kalaidzidis, L. Liang, J. Duncan, H. Shen, Y. Xu, K.E. Magnusson, J. Jalden, H.M. Blau, P. Paul-Gilloteaux, P. Roudot, C. Kervrann, F. Waharte, J.Y. Tinevez, S.L. Shorte, J. Willemse, K. Celler, G.P. van Wezel, H.W. Dan, Y.S. Tsai, C. Ortiz de Solorzano, J.C. Olivo-Marin, E. Meijering, Objective comparison of particle tracking methods, Nat. Methods 11 (2014) 281-289.

[143] E. Betzig, G.H. Patterson, R. Sougrat, O.W. Lindwasser, S. Olenych, J.S. Bonifacino, M.W. Davidson, J. Lippincott-Schwartz, H.F. Hess, Imaging intracellular fluorescent proteins at nanometer resolution, Science 313 (2006) 1642-1645.

[144] S.T. Hess, T.P. Girirajan, M.D. Mason, Ultra-high resolution imaging by fluorescence photoactivation localization microscopy, Biophys. J. 91 (2006) 4258-4272.

[145] M.J. Rust, M. Bates, X. Zhuang, Sub-diffraction-limit imaging by stochastic optical reconstruction microscopy (STORM), Nat. Methods 3 (2006) 793-795.

[146] K. Jaqaman, D. Loerke, M. Mettlen, H. Kuwata, S. Grinstein, S.L. Schmid, G. Danuser, Robust single-particle tracking in live-cell time-lapse sequences, Nat. Methods 5 (2008) 695-702.

[147] A. Serge, N. Bertaux, H. Rigneault, D. Marguet, Dynamic multiple-target tracing to probe spatiotemporal cartography of cell membranes, Nat. Methods 5 (2008) 687-694.

[148] J.G. Ritter, R. Veith, J.P. Siebrasse, U. Kubitscheck, High-contrast single-particle tracking by selective focal plane illumination microscopy, Opt. Express 16 (2008) 7142-7152.

[149] H.P. Kao, A.S. Verkman, Tracking of single fluorescent particles in three dimensions: use of cylindrical optics to encode particle position, Biophys. J. 67 (1994) 1291-1300.
[150] M.F. Juette, J. Bewersdorf, Three-dimensional tracking of single fluorescent particles with submillisecond temporal resolution, Nano Lett. 10 (2010) 4657-4663.

[151] Y. Wu, A. Ghitani, R. Christensen, A. Santella, Z. Du, G. Rondeau, Z. Bao, D. ColonRamos, H. Shroff, Inverted selective plane illumination microscopy (iSPIM) enables coupled cell identity lineaging and neurodevelopmental imaging in Caenorhabditis elegans, Proc. Natl. Acad. Sci. U. S. A. 108 (2011) 17708-17713.

[152] J.G. Ritter, R. Veith, A. Veenendaal, J.P. Siebrasse, U. Kubitscheck, Light sheet microscopy for single molecule tracking in living tissue, PLoS One 5 (2010) e11639.

[153] H. Deschout, K. Raemdonck, S. Stremersch, P. Maoddi, G. Mernier, P. Renaud, S. Jiguet, A. Hendrix, M. Bracke, R. Van den Broecke, M. Roding, M. Rudemo, J Demeester, S.C. De Smedt, F. Strubbe, K. Neyts, K. Braeckmans, On-chip light sheet illumination enables diagnostic size and concentration measurements of membrane vesicles in biofluids, Nanoscale 6 (2014) 1741-1747.

[154] N. Ruthardt, D.C. Lamb, C. Brauchle, Single-particle tracking as a quantitative microscopy-based approach to unravel cell entry mechanisms of viruses and pharmaceutical nanoparticles, Mol. Ther. 19 (2011) 1199-1211.

[155] H.C. Berg, How to track bacteria, Rev. Sci. Instrum. 42 (1971) 868-871.

[156] N.F. Reuel, A. Dupont, O. Thouvenin, D.C. Lamb, M.S. Strano, Three-dimensiona tracking of carbon nanotubes within living cells, ACS Nano 6 (2012) 5420-5428.

[157] S.R. Pavani, R. Piestun, Three dimensional tracking of fluorescent microparticles using a photon-limited double-helix response system, Opt. Express 16 (2008) 22048-22057.

[158] M.A. Thompson, M.D. Lew, M. Badieirostami, W.E. Moerner, Localizing and tracking single nanoscale emitters in three dimensions with high spatiotemporal resolution using a double-helix point spread function, Nano Lett. 10 (2010) 211-218.

[159] P. Prabhat, S. Ram, E.S. Ward, R.J. Ober, Simultaneous imaging of different focal planes in fluorescence microscopy for the study of cellular dynamics in three dimensions, IEEE Trans. Nanobioscience 3 (2004) 237-242.

[160] E. Toprak, H. Balci, B.H. Blehm, P.R. Selvin, Three-dimensional particle tracking via bifocal imaging, Nano Lett. 7 (2007) 2043-2045.

[161] H. Deschout, K. Raemdonck, J. Demeester, S.C. De Smedt, K. Braeckmans, FRAP in pharmaceutical research: practical guidelines and applications in drug delivery, Pharm. Res. 31 (2014) 255-270.

[162] T. Hothorn, F. Leisch, Case studies in reproducibility, Brief. Bioinform. 12 (2011) 288-300.

[163] R.D. Peng, Reproducible research in computational science, Science 334 (2011) 1226-1227.

[164] V.A. Fusaro, P. Patil, E. Gafni, D.P. Wall, P.J. Tonellato, Biomedical cloud computing with Amazon Web Services, PLoS Comput. Biol. 7 (2011) e1002147.

[165] Y. Han, A.M. Alsayed, M. Nobili, J. Zhang, T.C. Lubensky, A.G. Yodh, Brownian motion of an ellipsoid, Science 314 (2006) 626-630.

[166] S. Barua, J.W. Yoo, P. Kolhar, A. Wakankar, Y.R. Gokarn, S. Mitragotri, Particle shape enhances specificity of antibody-displaying nanoparticles, Proc. Natl. Acad. Sci. U. S. A. 110 (2013) 3270-3275.

[167] J.A. Champion, Y.K. Katare, S. Mitragotri, Particle shape: a new design parameter for micro- and nanoscale drug delivery carriers, J. Control. Release 121 (2007) 3-9.

[168] S.E. Birket, K.K. Chu, L. Liu, G.H. Houser, B.J. Diephuis, E.J. Wilsterman, G. Dierksen, M. Mazur, S. Shastry, Y. Li, J.D. Watson, A.T. Smith, B.S. Schuster, J. Hanes, W.E. Grizzle, E.J. Sorscher, G.J. Tearney, S.M. Rowe, A functional anatomic defect of the cystic fibrosis airway, Am. J. Respir. Crit. Care Med. 190 (2014) 421-432.

[169] L. Liu, K.K. Chu, G.H. Houser, B.J. Diephuis, Y. Li, E.J. Wilsterman, S. Shastry, G. Dierksen, S.E. Birket, M. Mazur, S. Byan-Parker, W.E. Grizzle, E.J. Sorscher, S.M. Rowe, G.J. Tearney, Method for quantitative study of airway functional microanatomy using micro-optical coherence tomography, PLoS One 8 (2013) e54473. 